\begin{tabular}{|c|c|}
\hline Title & $\begin{array}{l}\text { Photosensitized Intramolecular [2+2] Cycloaddition of } 1 \mathrm{H} \text {-Pyrrolo[2,3-b]pyridines Enabled by the A ssistance of Lewis } \\
\text { A cids }\end{array}$ \\
\hline Author(s) & A rai, Noriyoshi; Ohkuma, Takeshi \\
\hline Citation & $\begin{array}{l}\text { Journal of organic chemistry, 85(23), 15717-15725 } \\
\text { https://doi.org/10.1021/acs.joc.0c02231 }\end{array}$ \\
\hline Issue Date & $2020-12-04$ \\
\hline Doc URL & http:/hdl.handle.net/2115/83422 \\
\hline Rights & $\begin{array}{l}\text { This document is the unedited A uthor' sversion of a Submitted Work that was subsequently accepted for publication } \\
\text { in Journal of organic chemistry, copyright c A merican Chemical Society after peer review. To access the final edited } \\
\text { and published work see https:/pubs.acs.org/doi/10.1021/acs.joc.0c02231. }\end{array}$ \\
\hline Tyре & article (author version) \\
\hline File Information & jo-2020-02231k_revised2.pdf \\
\hline
\end{tabular}

Instructions for use 


\title{
Photosensitized Intramolecular [2+2] Cycloaddition of 1H-Pyrrolo[2,3- b]pyridines Enabled by the Assistance of Lewis Acids
}

\author{
Noriyoshi Arai, ${ }^{*}$ Takeshi Ohkuma*
}

Supporting Information Placeholder
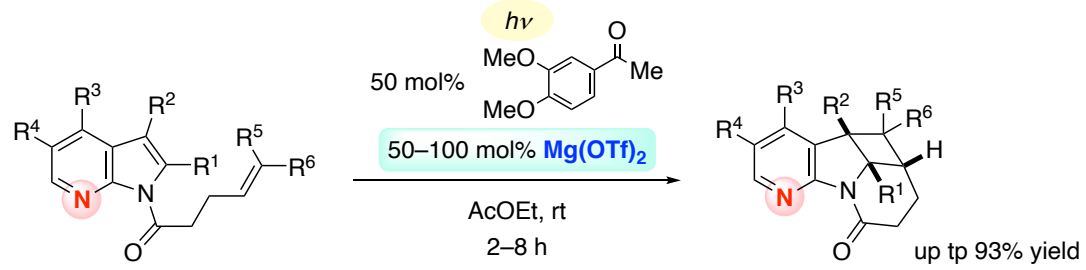

\begin{abstract}
The [2+2] photocycloaddition of alkenyl-tethered $1 H$-pyrrolo[2,3-b]pyridine derivatives sensitized with 3',4'dimethoxyacetophenone under irradiation by a high-pressure mercury lamp through Pyrex glass was dramatically accelerated by the addition of Lewis acids, preferably $\mathrm{Mg}(\mathrm{OTf})_{2}$, to give the products stereoselectively in high yields. The reaction without a Lewis acid gave only small amounts of the [2+2] cycloaddition products. Conformational fixation of the substrates by coordination with a Lewis acid was presumed to facilitate the cycloaddition.
\end{abstract}

$1 H$-Pyrrolo[2,3-b]pyridine (7-azaindole) derivatives are common structural motifs in many bioactive natural products, useful pharmaceuticals, and agrochemicals. ${ }^{1-5}$ On the other hand, in conjunction with a recent trend in drug discovery, i.e., "escape from flatland" concept ${ }^{6}$ conformationally restricted small-ring-fused compounds have also attracted much attention in modern medicinal chemistry. ${ }^{7,8}$ Cyclobutane-fused compounds form an important core of such compounds because of the balance they strike between the inherent rigidity of a small-ring compound and the greater stability toward ring-opening reactions compared with cyclopropane derivatives. ${ }^{9}$ Accordingly, novel cyclobutane-fused $1 \mathrm{H}$-pyrrolo[2,3$b]$ pyridine frameworks may be of interest in bioactive screening for new pharmaceutical candidates.

The photochemical [2+2] cycloaddition reaction is one of the most popular and straightforward methods of constructing 4-membered cyclic compounds, including cyclobutanes. ${ }^{10}$ In the course of our investigation on the photochemistry of 5membered heteroaromatic compounds, ${ }^{11}$ we recently reported the photochemical intramolecular [2+2] cycloaddition of allene-tethered indole derivatives sensitized by $3^{\prime}, 4^{\prime}-$ dimethoxyacetophenone giving methylenecyclobutane-fused indolines in a highly stereoselective manner (Scheme $1, \mathrm{a}){ }^{12}$ Prompted by these successful results, we initially attempted a reaction with allene-tethered $1 H$-pyrrolo[2,3-b]pyridine by simply applying the previous reaction conditions. Unexpected$1 y$, the reaction did not give any amount of the desired [2+2] addition product, but rather yielded unreacted material accompanied by small amounts of decomposed compounds including de-acylated $1 H$-pyrrolo[2,3-b]pyridine (Scheme 1, b).

Scheme 1. Construction of Cyclobutane-fused Nitrogen Heterocycles

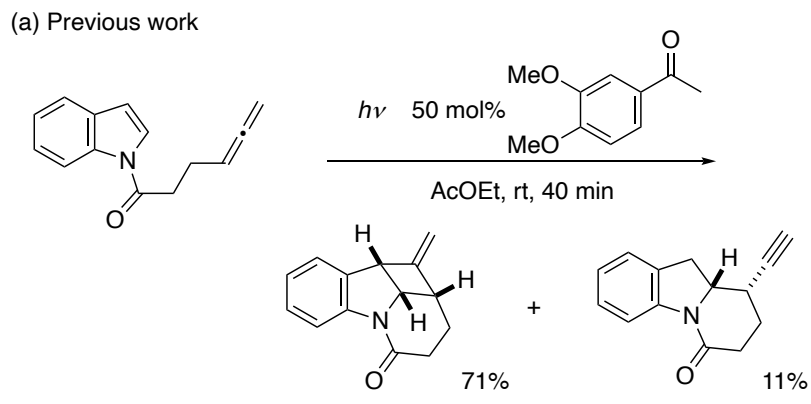

(b) Initial trial
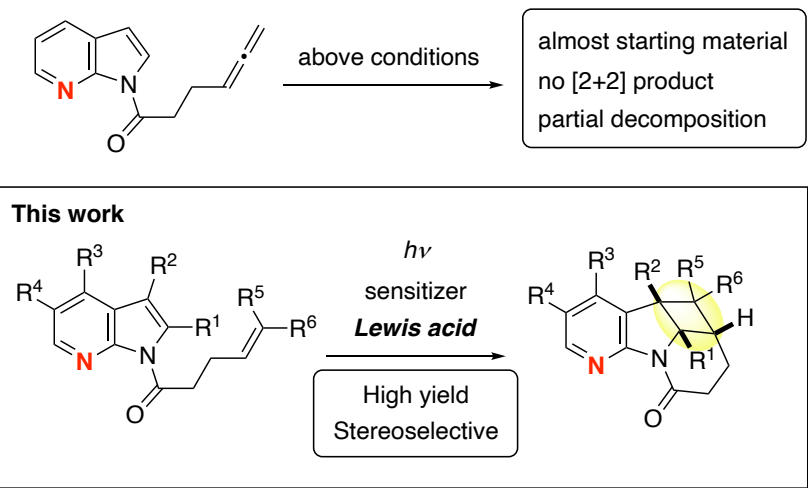

Actually, a bibliographic search revealed that the photochemical reaction using an excited state of $1 H$-pyrrolo[2,3$b]$ pyridines has scarcely been explored, and synthetic application of their photoreaction is relatively unknown. ${ }^{13,14}$ To the best of our knowledge, the Paternò-Büchi type oxetane formation with low efficiency has been the only example of fused 4-membered ring formation through the photochemical reac- 
tion of $1 H$-pyrrolo[2,3-b]pyridines for a long time. ${ }^{13,15}$ Quite recently, You and collaborators have reported similar intramolecular cyclization of $1 H$-pyrrolo[2,3-b]pyridines that likely have relatively low $\Delta G\left(\mathrm{~T}_{1}-\mathrm{S}_{0}\right)$ values sensitized by Ir visiblelight photocatalyst. ${ }^{16}$ Independently, Fu and collaborators have reported an excellent work dealing with similar cycloaddition. ${ }^{17}$ This background prompted us to develop new, synthetically useful photochemical reactions of $1 H$-pyrrolo[2,3$b]$ pyridines represented by the [2+2] cycloaddition reaction. We report herein the novel photocyclization of $1 \mathrm{H}$ pyrrolo[2,3- $b]$ pyridines derivatives to afford cyclobutanefused tetracyclic frameworks in a highly stereoselective manner.

At the early stage of our investigation, we envisaged that the repulsion between the lone pair of the pyridine nitrogen and the side chain would hold the alkene moiety in an unfavorable conformation for the intramolecular cyclization. To circumvent this problem, we attempted a reaction in the presence of a Lewis acid in addition to the originally optimized conditions for indoles, ${ }^{12 a}$ expecting that coordination of the Lewis acid to the pyridine nitrogen and amide carbonyl would bring the alkenyl moiety close to the $\mathrm{C} 2-\mathrm{C} 3$ bond.

We chose 1-( $\omega$-alkenoyl)-1H-pyrrolo[2,3-b]pyridine derivative 1a instead of the above-mentioned allene derivative in order to simplify the reaction system and to facilitate the product study. Referring to our previous result, ${ }^{12 a}$ we carried out reactions of 1a sensitized by 3',4'-dimethoxyacetophenone (3, $50 \mathrm{~mol} \%$ ) in ethyl acetate (degassed by freeze-thaw cycles before use) under irradiation by a high-pressure mercury lamp through Pyrex glass in the presence of a series of Lewis acids (Table 1). Metal triflates were chosen as candidates because of the balance of Lewis acidity and solubility in ethyl acetate. The reaction was discontinued in $3 \mathrm{~h}$ irrespective of the consumption of $\mathbf{1 a}$.

Table 1. Screening of Lewis Acids ${ }^{a}$

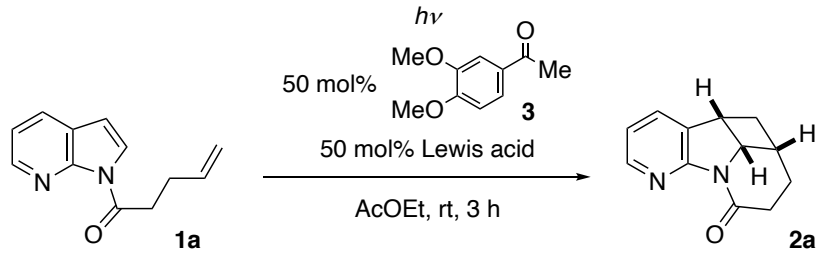

\begin{tabular}{|l|l|l|l|}
\hline entry & Lewis acid & conv. $(\%)^{b}$ & 2a $(\%)^{b, c}$ \\
\hline 1 & $\mathrm{Al}(\mathrm{OTf})_{3}$ & 94 & $82(87)$ \\
\hline 2 & $\mathrm{Mg}(\mathrm{OTf})_{2}$ & 82 & $70(85)$ \\
\hline 3 & $\mathrm{Mg}(\mathrm{OTf})_{2}{ }^{d}$ & $>99$ & $92(92)$ \\
\hline 4 & $\mathrm{Yb}(\mathrm{OTf})_{3}$ & 80 & $74(93)$ \\
\hline 5 & $\mathrm{Bi}(\mathrm{OTf})_{3}$ & 89 & $75(84)$ \\
\hline 6 & $\mathrm{Ca}(\mathrm{OTf})_{2}$ & 50 & $38(76)$ \\
\hline 7 & $\mathrm{Sc}(\mathrm{OTf})_{3}$ & 62 & $33(53)$ \\
\hline 8 & $\mathrm{LiOTf}^{d}$ & 32 & $20(63)$ \\
\hline 9 & $(+)-\mathrm{CSA}^{d}$ & 26 & $9(35)$ \\
\hline 10 & $\mathrm{H}_{3} \mathrm{PO}_{4}{ }^{d}$ & 17 & $2(12)$ \\
\hline 11 & none $^{2}$ & 18 & $6(33)$ \\
\hline
\end{tabular}

${ }^{a}$ All reactions were carried out in a Pyrex test tube by external irradiation with a high-pressure $\mathrm{Hg}$ lamp at a concentration of 10 $\mathrm{mM}$ using $0.1 \mathrm{mmol}$ of $\mathbf{1 a} .{ }^{b}$ Determined by the ${ }^{1} \mathrm{H}$ NMR integral ratio using triphenylmethane as an internal standard. ${ }^{c}$ Yields based on the recovered starting material are shown in parentheses. ${ }^{d} 100 \mathrm{~mol} \%$.

The yield of $\mathbf{2 a}$ was largely dependent on the choice of an additive. The reaction proceeded well in the presence of $\mathrm{Al}$, $\mathrm{Mg}, \mathrm{Yb}$, and Bi triflates, giving the tetracyclic product $\mathbf{2 a}$ in moderate or high yields (entries 1-5), whereas the $\mathrm{Ca}$ and $\mathrm{Sc}$ triflates gave considerably inferior conversion (entries 6 and 7). The relative configuration of $\mathbf{2} \mathbf{a}$ was determined by a conventional NOE experiment (see the Supporting Information). LiOTf, a triflate of the group 1 elements, showed a much less pronounced promoting effect (entry 8). A Brønsted acid, camphorsulfonic acid, exhibited almost no positive effect (entry 9). Phosphoric acid, which has a lower acidity than CSA, gave much inferior result (entry 10). In this case, the reaction mixture got intensely cloudy. The low conversion and yield might be attributable to the decreased transmittance. When the reaction was carried out in the absence of additive, 2a was obtained in only $6 \%$ yield accompanied by comparable amounts of acyl-cleaved by-products (entry 11). In cases in which the material balance was not satisfactory, formation of a yellowcolored unidentified component tended to be observed. A reaction with $100 \mathrm{~mol} \% \mathrm{Mg}(\mathrm{OTf})_{2}$ afforded almost complete conversion with negligible formation of the colored materials (entry 3 ). It should be noted that ring junctures were created with perfect diastereoselectivity. The presence of the sensitizer 3 was indispensable for this reaction. This is because, in the absence of $\mathbf{3}$, non-productive decomposition of 1a occurred competitively, causing a considerable decrease of the yield of $\mathbf{2 a}$, because the absorption edge of $\mathbf{1 a}$ reached into the irradiation range (approximately $>300 \mathrm{~nm}$ ). The product $\mathbf{2 a}$ was stable under simple irradiation and recovered almost quantitatively after 3 h. However, when isolated $\mathbf{2 a}$ was irradiated under the same conditions as in entry 3 , slight decomposition was observed ( $85 \%$ recovery).

Choosing $\mathrm{Al}(\mathrm{OTf})_{3}, \mathrm{Mg}(\mathrm{OTf})_{2}$, and $\mathrm{Yb}(\mathrm{OTf})_{3}$ as promising candidates, we adjusted the reaction conditions in more detail to maximize the yield of $\mathbf{2 a}$ (Table 2). ${ }^{18}$ The reactions shown in Table 2 were carried out in a photochemical reaction vessel for internal irradiation by using $100 \mathrm{~mol} \%$ of Lewis acid. The reactions were discontinued as soon as possible after consumption of the starting materials ( $>99 \%$ conversion). $\mathrm{Al}(\mathrm{OTf})_{3}$ and $\mathrm{Mg}(\mathrm{OTf})_{2}$ gave comparable results. The reactions were complete within $2 \mathrm{~h}$, affording $\mathbf{2 a}$ in high yields (entries 1 and 2). The slight difference in the yields was attributable to the formation of decomposed materials. The reaction scale could be increased without problem to afford the product in high yield (entry 3 ). The reaction with $\mathrm{Yb}(\mathrm{OTf})_{3}$ was considerably slower than the reactions using the above two conditions, though the yield of $\mathbf{2 a}$ was also high and the reaction was clean (entry 4). Thus, we set $\mathrm{Mg}(\mathrm{OTf})_{2}$ as the best Lewis acid for this reaction. ${ }^{19}$

Table 2. Maximizing the Product Yield ${ }^{a}$ 

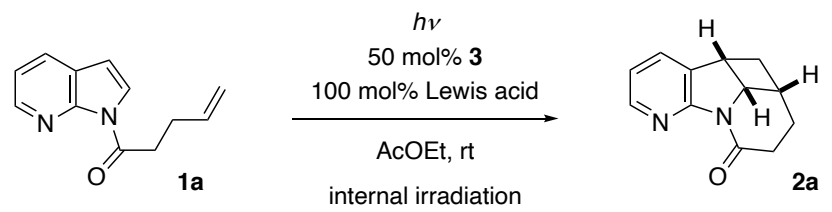

\begin{tabular}{|l|l|l|l|}
\hline entry & Lewis acid & irradiation time $(\mathrm{h})$ & $\mathbf{2 a}(\%)^{b}$ \\
\hline 1 & $\mathrm{Al}(\mathrm{OTf})_{3}$ & 2 & 89 \\
\hline 2 & $\mathrm{Mg}(\mathrm{OTf})_{2}$ & 2 & 93 \\
\hline $3^{c}$ & $\mathrm{Mg}(\mathrm{OTf})_{2}$ & 2 & 89 \\
\hline 4 & $\mathrm{Yb}(\mathrm{OTf})_{3}$ & 8 & 90 \\
\hline
\end{tabular}

${ }^{a}$ All reactions were carried out in a Pyrex reaction vessel for photochemical reaction by internal irradiation with a high-pressure Hg lamp at a concentration of $10 \mathrm{mM}$ using $0.2 \mathrm{mmol}$ of 1a. ${ }^{b}$

Yields of isolated product. ${ }^{c}$ A preparative scale $(1 \mathrm{mmol})$ reaction.

With the optimized conditions in hand, we explored the reaction using a variety of $1 H$-pyrrolo[2,3-b]pyridine derivatives (Scheme 2). The substituents at the pyridine ring of the substrates showed significant influence on the reaction. The reactions of $\mathbf{1 b}(4-\mathrm{Cl})$ and $1 \mathbf{c}\left(5-\mathrm{CO}_{2} \mathrm{Me}\right)$, in which the pyridine ring was substituted by an electron-withdrawing group, were considerably slower than that of 1a, giving moderate yields of the cyclized products $\mathbf{2 b}$ and $\mathbf{2 c}$, respectively. On the other hand, in the case of $1 \mathbf{d}(4-\mathrm{MeO})$, in which the pyridine ring was substituted by an electron-donating group, the reaction proceeded as rapidly as that of $\mathbf{1 a}$, affording the product $\mathbf{2 d}$ in high yield. The reaction of 1e (5-BocNMe) gave 2 e in $77 \%$ yield, though the progress was slow. Introduction of a methyl group at the 3-position (1f) gave almost the same result as 1a, while Me-substitution at the 2-position retarded the reaction, giving the product $\mathbf{2 g}$ in moderate yield. Irradiation of the 2,3diMe-substituted substrate $\mathbf{1 h}$ afforded the [2+2] adduct with adjacent quaternary carbons $\mathbf{2 h}$ in spite of severe steric hindrance. In this case, most of the $\mathbf{1 h}$ was consumed and the rest of the materials were not identifiable, presumably due to undesirable decomposition reactions such as deacylation and acylmigration. ${ }^{14,20}$ In all cases, the product was obtained as a single diastereomer. This suggests the synthetic potential of this reaction for the stereoselective preparation of $1 H$-pyrrolo[2,3$b]$ pyridines with fused rings.

\section{Scheme 2. Substrate Scope $e^{a, b}$}

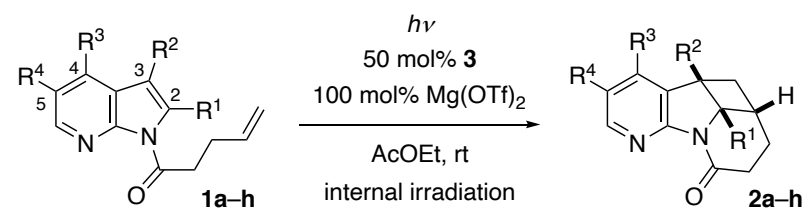

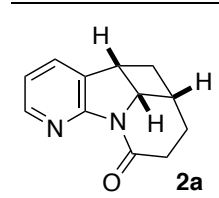<smiles>[213CH3]</smiles><smiles>O=C1CC[C@H]2C[C@H]3c4c(Cl)ccnc4N1[C@H]23</smiles><smiles>COC(=O)c1cnc2c(c1)[C@H]1C[C@H]3CCC(=O)N2[C@@H]3C1</smiles><smiles>COc1ccnc2c1[C@H]1C[C@H]3CCC(=O)N2[C@@H]1C3</smiles>

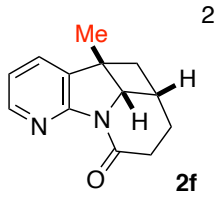

2 h, $88 \%$<smiles>[M]C1CCC(=O)N2c3ncccc3[C@H]([R10])C[C@H]12</smiles>

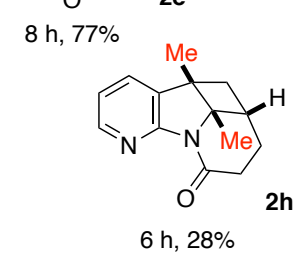

${ }^{a}$ All reactions were carried out in a Pyrex reaction vessel for photochemical reaction by internal irradiation with a highpressure $\mathrm{Hg}$ lamp at a concentration of $10 \mathrm{mM}$ using $0.2 \mathrm{mmol}$ of 1a. ${ }^{b}$ Yields of isolated product.

This reaction is also applicable to substrates that have a substituted alkene moiety at the linker terminal (Scheme 3). When the terminal allenyl substrate $\mathbf{1 i}\left(\mathrm{R}^{5}, \mathrm{R}^{6}:=\mathrm{CH}_{2}\right)$, in which we failed initially, was irradiated under the same conditions as above, the methylenecyclobutane-fused product $\mathbf{2 i}$ was obtained in moderate yield, accompanied by small amounts of terminal alkyne $\mathbf{4}$. Compound $\mathbf{4}$ was likely produced through 1,5-hydrogen transfer of the biradical intermediate. ${ }^{12 a}$ Irradiation of a substrate with two terminal methyl groups $1 \mathbf{j}\left(\mathrm{R}^{5}, \mathrm{R}^{6}\right.$ : $\mathrm{Me})$ gave the [2+2] adduct $\mathbf{2 \mathbf { j }}$ in $\mathbf{7 5 \%}$ yield. Also in this case, the tricyclic by-product 5 , which was presumed to come from 1,5-hydrogen transfer, was obtained. The reaction of the substrate with the cyclohexenyl moiety $\mathbf{1 k}$ afforded the pentacyclic $1 H$-pyrrolo[2,3-b]pyridine derivatives $2 \mathbf{k a}$ and $\mathbf{2} \mathbf{k b}$. Stereoselectivity around the cyclobutane ring was also perfect in this case, though the stereochemical control adjacent to the cyclobutane moiety was incomplete. Unexpectedly, tetracyclic ester 6 was isolated after chromatographic purification. We surmised that the ester $\mathbf{6}$ was formed via methanolysis of $\mathbf{2} \mathbf{k a}$ during preparative TLC development with chloroformmethanol, probably due to the intrinsic strain of $2 \mathbf{k a}$.

Scheme 3. Variety of the Alkene Moiety ${ }^{a, b}$ 

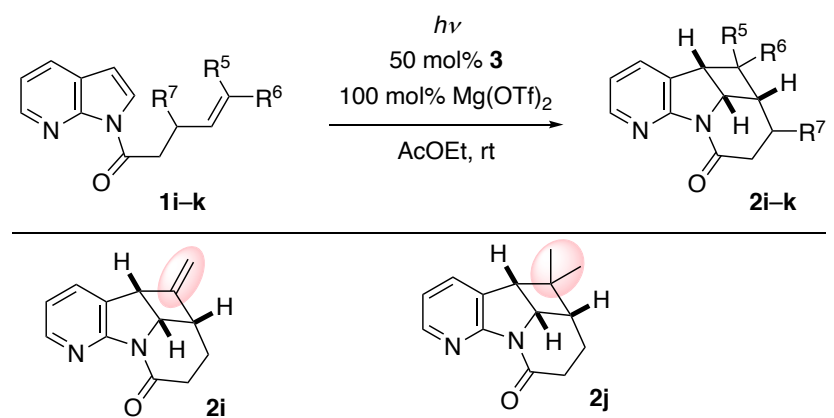

$2 \mathrm{~h}, 66 \%$

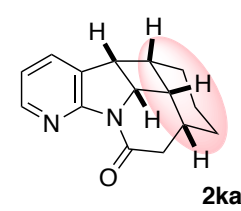

$3 \mathrm{~h}, 75 \%$

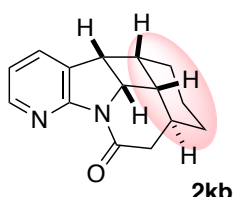

$2 \mathrm{~h}, 68 \%, 9 \%$

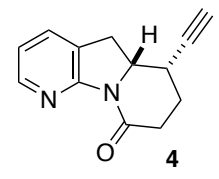

$12 \%$
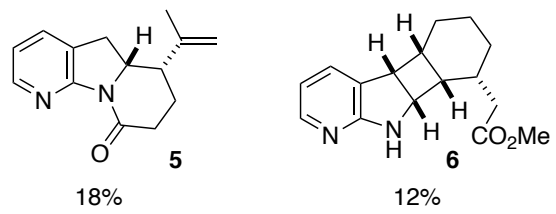

a All reactions were carried out in a Pyrex reaction vessel for photochemical reaction by internal irradiation with a highpressure $\mathrm{Hg}$ lamp at a concentration of $10 \mathrm{mM}$ using $0.2 \mathrm{mmol}$ of 1a. ${ }^{b}$ Yields of isolated product.

As shown above, the presence of a Lewis acid, preferably $\mathrm{Mg}(\mathrm{OTf})_{2}$, is indispensable for obtaining the [2+2] cycloaddition products in good yields. Although the role of magnesium was not fully disclosed at this stage, we presumed that the precoordination of the $1 H$-pyrrolo[2,3-b]pyridine substrates to magnesium would bring two reaction sites in the molecule closer and promote the intramolecular [2+2] cycloaddition based on the following.

First, a conformational analysis of 1-acyl- $1 H$-pyrrolo[2,3$b]$ pyridine 1a and the corresponding indole derivative at the ground state was carried out by using theoretical calculation. ${ }^{21}$ In the case of $\mathbf{1 a}$, the conformer $\mathbf{1} \mathbf{a}_{\mathrm{CO} \text {-out, }}$ in which the alkene moiety and C2 position is more distant, is more stable than $\mathbf{1} \mathbf{a}_{\mathrm{CO}-\text {-in }}$ by $2-3 \mathrm{kcal} / \mathrm{mol}$ considering that many side-chain conformers exist in narrow differences in energy. On the contrary, in the case of the indole derivative, the conformer Ind $_{\mathrm{CO} \text {-in, }}$ which is preferable for the cycloaddition, is more stable than Ind $_{\text {CO-out }}$ by about $2 \mathrm{kcal} / \mathrm{mol}$ (Figure 1). This result is consistent with the experimental results shown in Scheme 1 and Table 1.

Figure 1. Comparison of conformers between $1 \mathrm{a}$ and the Corresponding Indole Derivative

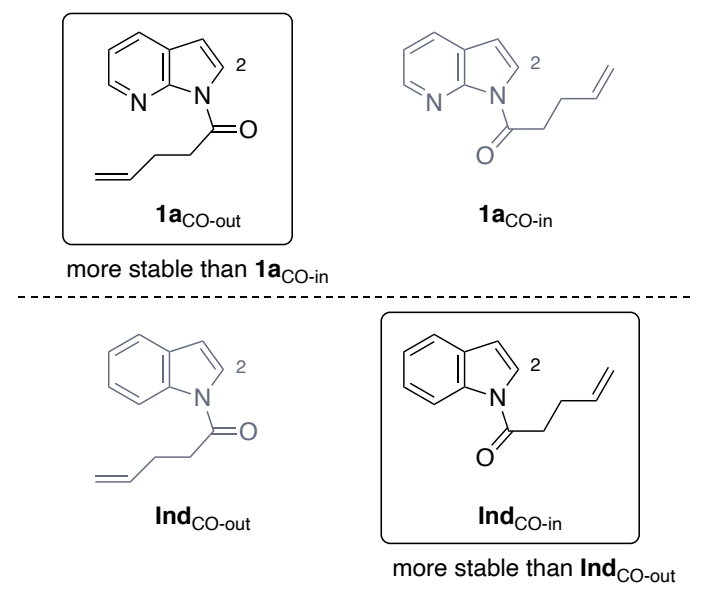

Second, when $\operatorname{Mg}(\mathrm{OTf})_{2}$ was added to a solution of 1a in acetone- $d_{6}$ in several portions, the ${ }^{1} \mathrm{H}$ NMR signals around pyridine nitrogen and the amide moiety showed a significant shift in proportion to the added amount of $\mathrm{Mg}(\mathrm{OTf})_{2}$ (Figure S1). On the other hand, the same NMR experiment using the sensitizer $\mathbf{3}$ instead of $\mathbf{1 a}$ gave no recognizable change in the ${ }^{1} \mathrm{H}$ NMR spectrum (Figure S2). This result clearly shows that $\mathrm{Mg}(\mathrm{OTf})_{2}$ has apparent coordination with 1a only by mixing and shows no apparent coordination with the sensitizer 3 .

However, the possibility that the coordination facilitated a photochemical process, such as energy transfer, ${ }^{17,22}$ is also conceivable. The triplet energy $\left(E_{\mathrm{T}}\right)$ of $\mathbf{3}$ is reported as $E_{\mathrm{T}}=67.3 \mathrm{kcal} / \mathrm{mol}^{23}$ Though we could not find $E_{\mathrm{T}}$ of $N$-acyl$1 H$-pyrrolo[2,3-b]pyridines in the literature, energy transfer from ${ }^{3}[3]^{*}$ to both of coordinated and non-coordinated substrates seems possible, because a similar compound can be sensitized by an Ir photocatalyst whose $E_{\mathrm{T}}$ is $61.2 \mathrm{kcal} / \mathrm{mol} .{ }^{17}$ We found that the addition of $\operatorname{Mg}(\mathrm{OTf})_{2}$ hardly affected the reaction of compound 7 with an alkenyl linker at the 3position, whose conformation is not under the influence of the pyridine nitrogen, and the conformational fixation was not necessary (Scheme 4), though the possibility that the 5-exo cyclization of 7 is easier than the corresponding 6-exo cyclization of 1 a could not be ruled out.

\section{Scheme 4. Reaction of 3-Substituted Compound 7}<smiles>C=CCC(Cc1ccccc1)(Cc1cn(C(C)=O)c2ncccc12)C(=O)OCC</smiles>

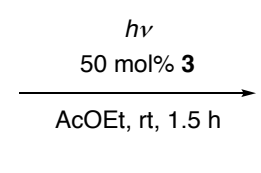

$\mathrm{Mg}(\mathrm{OTf})$

$100 \mathrm{~mol} \% \quad 88 \%$ none $\quad 92 \%$
Reflecting on these results and the findings from previous studies, ${ }^{17,24,25}$ we presumed that the mechanism underlying this reaction was as shown in Scheme 5. The triplet excited state of 3 is generated by the irradiation, transferring the energy to Mg-coordinated 1a, which has the conformation preferable for the intramolecular cyclization, to produce its triplet state ${ }^{3}\left[\mathbf{1} \mathbf{a}+\mathrm{Mg}^{2+}\right]^{*}$. This species undergoes stepwise bond-forming 
reaction to afford the $[2+2]$ cycloaddition product $\mathbf{2 a}$. Cycloaddition through direct excitation would be negligible because the reaction without $\mathbf{3}$ gave only small amounts of $\mathbf{2 a}$ with a partial decomposition of $\mathbf{1 a}$ (Scheme S1).

\section{Scheme 5. Plausible Reaction Pathway}

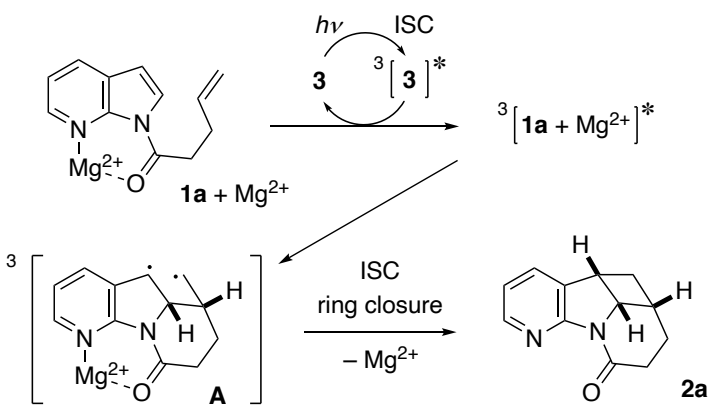

In conclusion, we have developed a method for the construction of cyclobutane-fused tetracyclic $1 \mathrm{H}$-pyrrolo[2,3$b]$ pyridine frameworks through the $[2+2]$ photochemical cycloaddition reaction under the sensitization with $3^{\prime}, 4^{\prime}$ dimethoxylacetophenone. This reaction provides an efficient route to unique nitrogen heterocyclic scaffolds that are otherwise difficult to prepare, generating molecular complexity from readily available starting materials.

\section{EXPERIMENTAL SECTION}

General remarks. The photochemical reactions were carried out with a RIKO UVL 100-HA high-pressure mercury lamp covered with a Pyrex cooling jacket whose transmittance is $25 \%$ at $300 \mathrm{~nm}, 70 \%$ at $320 \mathrm{~nm},>90 \%$ over $360 \mathrm{~nm}$. The emission wave length ranges from $312 \mathrm{~nm}$ to $577 \mathrm{~nm}$ and 365 $\mathrm{nm}$ light is most strongly emitted. The emission property of the light source is shown in the Supporting Information (Figure S3). The distance from the light source to the reaction vessel is $30 \mathrm{~mm}$ for the external irradiation and $25 \mathrm{~mm}$ for the internal irradiation (center-to-center). NMR spectra were obtained on a JEOL JNM-ECS400 spectrometer. Carbon multiplicity was assigned by a DEPT experiment and described as follows: methyl, $\mathrm{CH}_{3}$; methylene, $\mathrm{CH}_{2}$; methine, $\mathrm{CH}$; quaternary, C. IR spectra were recorded on a JASCO FT/IR-4100 spectrophotometer. UV spectra were obtained on a JASCO UV/Vis spectrophotometer Ubest-30. Silica gel column chromatography was performed with Fuji Silysia PSQ60B or FL60D. Preparative thin-layer chromatography (TLC) was carried out with Wako Gel B-5F (FUJIFILM Wako Pure Chemical Corporation). HPLC separation was performed using a JASCO HPLC system comprised of PU-2080 Plus, DG2080-53, UV-2075 Plus, and CO-2065. Solvents and reagents were used as received unless otherwise noted. Mass spectrometry and elemental analysis were carried out at the Instrumental Analysis Division, Global Facility Center, Creative Research Institution, Hokkaido University.

Preparation and physical data of the starting materials. The starting materials were prepared by condensation between $1 H$-pyrrolo[2,3-b]pyridines and alkenyl carboxylic acids based on the method reported by Tokuyama and collaborators. ${ }^{26}$ Some carboxylic acids are commercially available and other known compounds were prepared according to a procedure described in the literature. ${ }^{27,28}$ The physical data of new compounds are as follows:

1-(1H-Pyrrolo[2,3-b]pyridin-1-yl)pent-4-en-1-one (1a): Yield: $0.970 \mathrm{~g}(4.84 \mathrm{mmol}, 95 \%)$ from pent-4-enoic acid $(0.509 \mathrm{~g}, 5.08 \mathrm{mmol})$. Colorless oil. UV-Vis $\left(1.6 \times 10^{-4} \mathrm{~mol} / \mathrm{L}\right.$, ethyl acetate) $\lambda_{\max } 292 \mathrm{~nm}\left(\varepsilon 3.2 \times 10^{3}\right.$, shoulder). IR (KBr) 3148, 3077, 2979, 2921, 1712, 1580, 1530, 1408, 1297, 1263, $1205,1057,988,915,890,803,779,735 \mathrm{~cm}^{-1} .{ }^{1} \mathrm{H}$ NMR $(400$ $\left.\mathrm{MHz}, \mathrm{CDCl}_{3}\right) \delta 8.37(\mathrm{dd}, J=4.8,1.7 \mathrm{~Hz}, 1 \mathrm{H}), 8.00(\mathrm{~d}, J=4.1$ $\mathrm{Hz}, 1 \mathrm{H}), 7.89$ (dd, $J=7.8,1.7 \mathrm{~Hz}, 1 \mathrm{H}), 7.20$ (dd, $J=7.8,4.8 \mathrm{~Hz}$, $1 \mathrm{H}), 6.60(\mathrm{~d}, J=4.1 \mathrm{~Hz}, 1 \mathrm{H}), 6.04-5.94(\mathrm{~m}, 1 \mathrm{H}), 5.18-5.13(\mathrm{~m}$, $1 \mathrm{H}), 5.07-5.03(\mathrm{~m}, 1 \mathrm{H}), 3.68(\mathrm{t}, J=7.4 \mathrm{~Hz}, 2 \mathrm{H}), 2.64-2.58(\mathrm{~m}$, 2H). ${ }^{13} \mathrm{C}\left\{{ }^{1} \mathrm{H}\right\}$ NMR $\left(100 \mathrm{MHz}, \mathrm{CDCl}_{3}\right) \delta 171.4(\mathrm{C}), 147.6(\mathrm{C})$, $143.7(\mathrm{CH}), 137.1(\mathrm{CH}), 129.2(\mathrm{CH}), 125.4(\mathrm{CH}), 123.7(\mathrm{C})$, 118.6 (CH), $115.5\left(\mathrm{CH}_{2}\right), 105.6(\mathrm{CH}), 37.0\left(\mathrm{CH}_{2}\right), 28.4\left(\mathrm{CH}_{2}\right)$. HRMS (ESI-orbitrap) $m / z:[\mathrm{M}+\mathrm{Na}]^{+}$calcd for $\mathrm{C}_{12} \mathrm{H}_{12} \mathrm{~N}_{2} \mathrm{NaO}$, 223.0842; found, 223.0844.

1-(4-Chloro-1H-pyrrolo[2,3-b]pyridin-1-yl)pent-4-en-1one (1b): Yield: $111.6 \mathrm{mg}(0.476 \mathrm{mmol}, 94 \%)$ from pent-4enoic acid (50.5 mg, $0.504 \mathrm{mmol})$. Colorless solid. IR ( $\mathrm{KBr})$ 3082, 1708, 1567, 1526, 1383, 1362, 1284, 1268, 1242, 1186, 903, 840, 755, $729 \mathrm{~cm}^{-1} .{ }^{1} \mathrm{H}$ NMR (400 MHz, $\left.\mathrm{CDCl}_{3}\right) \delta 8.27$ $(\mathrm{d}, J=5.3 \mathrm{~Hz}, 1 \mathrm{H}), 8.04(\mathrm{~d}, J=4.1 \mathrm{~Hz}, 1 \mathrm{H}), 7.23(\mathrm{~d}, J=5.3 \mathrm{~Hz}$, $1 \mathrm{H}), 6.72(\mathrm{~d}, J=4.1 \mathrm{~Hz}, 1 \mathrm{H}), 6.02-5.92(\mathrm{~m}, 1 \mathrm{H}), 5.18-5.12(\mathrm{~m}$, 1H), 5.07-5.04 (m, 1H), 3.65 (t, $J=7.4 \mathrm{~Hz}, 2 \mathrm{H}), 2.63-2.57$ (m, $2 \mathrm{H}) .{ }^{13} \mathrm{C}\left\{{ }^{1} \mathrm{H}\right\}$ NMR $\left(100 \mathrm{MHz}, \mathrm{CDCl}_{3}\right) \delta 171.3(\mathrm{C}), 148.0(\mathrm{C})$, $144.2(\mathrm{CH}), 136.9(\mathrm{CH}), 136.4(\mathrm{C}), 126.0(\mathrm{CH}), 123.0(\mathrm{C})$, $118.7(\mathrm{CH}), 115.7\left(\mathrm{CH}_{2}\right), 103.7(\mathrm{CH}), 37.1\left(\mathrm{CH}_{2}\right), 28.4\left(\mathrm{CH}_{2}\right)$. HRMS (ESI-orbitrap) $m / z:[\mathrm{M}+\mathrm{Na}]^{+}$calcd for $\mathrm{C}_{12} \mathrm{H}_{11}{ }^{35} \mathrm{ClN}_{2} \mathrm{NaO}$, 257.0452; found, 257.0453.

Methyl 1-(pent-4-enoyl)-1 H-pyrrolo[2,3-b]pyridine-5carboxylate (1c): Yield: $77.5 \mathrm{mg}(0.300 \mathrm{mmol}, 76 \%)$ from pent-4-enoic acid (39.4 mg, $0.394 \mathrm{mmol})$. Colorless solid. IR (KBr) 3075, 2995, 2952, 1709, 1526, 1441, 1391, 1310, 1281, 1189, 986, 909, 772, 756, $744 \mathrm{~cm}^{-1} .{ }^{1} \mathrm{H}$ NMR (400 MHz, $\left.\mathrm{CDCl}_{3}\right) \delta 9.03(\mathrm{~d}, J=2.0 \mathrm{~Hz}, 1 \mathrm{H}), 8.52$ (d, $\left.J=2.0 \mathrm{~Hz}, 1 \mathrm{H}\right), 8.06$ (d, $J=4.1 \mathrm{~Hz}, 1 \mathrm{H}), 6.68(\mathrm{~d}, J=4.1 \mathrm{~Hz}, 1 \mathrm{H}), 6.03-5.93(\mathrm{~m}, 1 \mathrm{H})$, 5.19-5.13 (m, 1H), 5.08-5.04 (m, 1H), $3.98(\mathrm{~s}, 3 \mathrm{H}), 3.68(\mathrm{t}$, $J=7.4 \mathrm{~Hz}, 2 \mathrm{H}), 2.64-2.58(\mathrm{~m}, 2 \mathrm{H}) .{ }^{13} \mathrm{C}\left\{{ }^{1} \mathrm{H}\right\} \mathrm{NMR}(100 \mathrm{MHz}$, $\left.\mathrm{CDCl}_{3}\right) \delta 171.2(\mathrm{C}), 166.2(\mathrm{C}), 149.3(\mathrm{C}), 145.7(\mathrm{CH}), 136.8$ $(\mathrm{CH}), 130.9(\mathrm{CH}), 126.9(\mathrm{CH}), 123.1(\mathrm{C}), 121.2(\mathrm{C}), 115.7$ $\left(\mathrm{CH}_{2}\right), 106.0(\mathrm{CH}), 52.3\left(\mathrm{CH}_{3}\right), 37.2\left(\mathrm{CH}_{2}\right), 28.4\left(\mathrm{CH}_{2}\right)$. HRMS (ESI-orbitrap) $m / z:[\mathrm{M}+\mathrm{Na}]^{+}$calcd for $\mathrm{C}_{14} \mathrm{H}_{14} \mathrm{~N}_{2} \mathrm{NaO}_{3}$, 281.0897; found, 281.0899.

1-(4-Methoxy-1H-pyrrolo[2,3-b]pyridin-1-yl)pent-4-en1-one (1d): Yield: $137.8 \mathrm{mg}(0.598 \mathrm{mmol}, 88 \%)$ from pent-4enoic acid (68.0 mg, $0.679 \mathrm{mmol})$. Colorless solid. IR (KBr) 3120, 2976, 2947, 1700, 1604, 1579, 1527, 1496, 1411, 1361, 1288, 1229, 1064, 994, 918, 806, 786, $736 \mathrm{~cm}^{-1} .{ }^{1} \mathrm{H}$ NMR $\left(400 \mathrm{MHz}, \mathrm{CDCl}_{3}\right) \delta 8.26(\mathrm{~d}, J=5.6 \mathrm{~Hz}, 1 \mathrm{H}), 7.84(\mathrm{~d}, J=4.1 \mathrm{~Hz}$, $1 \mathrm{H}), 6.68-6.67(\mathrm{~m}, 2 \mathrm{H}), 6.03-5.93(\mathrm{~m}, 1 \mathrm{H}), 5.17-5.12(\mathrm{~m}$, $1 \mathrm{H}), 5.06-5.02(\mathrm{~m}, 1 \mathrm{H}), 3.99(\mathrm{~s}, 3 \mathrm{H}), 3.66(\mathrm{t}, J=7.3 \mathrm{~Hz}, 2 \mathrm{H})$, 2.62-2.56 (m, 2H). ${ }^{13} \mathrm{C}\left\{{ }^{1} \mathrm{H}\right\}$ NMR $\left(100 \mathrm{MHz}, \mathrm{CDCl}_{3}\right) \delta 171.7$ (C), $159.6(\mathrm{C}), 149.2(\mathrm{C}), 145.7(\mathrm{CH}), 137.2(\mathrm{CH}), 123.1(\mathrm{CH})$, $115.4\left(\mathrm{CH}_{2}\right), 113.6(\mathrm{C}), 102.8(\mathrm{CH}), 100.8(\mathrm{CH}), 55.5\left(\mathrm{CH}_{3}\right)$, $37.0\left(\mathrm{CH}_{2}\right), 28.5\left(\mathrm{CH}_{2}\right)$. HRMS (ESI-orbitrap) $m / z$ : $[\mathrm{M}+\mathrm{Na}]^{+}$ calcd for $\mathrm{C}_{13} \mathrm{H}_{14} \mathrm{~N}_{2} \mathrm{NaO}_{2}, 253.0948$; found, 253.0950 .

tert-Butyl $N$-methyl- $N$-[1-(pent-4-enoyl)-1 $H$-pyrrolo[2,3b]pyridin-5-yl]carbamate (1e): Yield: $13.0 \mathrm{mg}$ (0.0395 mmol, $49 \%$ ) from pent-4-enoic acid $(8.0 \mathrm{mg}, 0.0799 \mathrm{mmol})$. Pale yellow oil. IR (KBr) 3078, 2978, 2931, 1705, 1477, 1393, 
$1368,1308,1249,1202,1151,915,737 \mathrm{~cm}^{-1} .{ }^{1} \mathrm{H}$ NMR $(400$ $\left.\mathrm{MHz}, \mathrm{CDCl}_{3}\right) \delta 8.26$ (br d, $\left.J=2.0 \mathrm{~Hz}, 1 \mathrm{H}\right), 8.00(\mathrm{~d}, J=4.1 \mathrm{~Hz}$, $1 \mathrm{H}), 7.74$ (br s, 1H), 6.57 (d, $J=4.1 \mathrm{~Hz}, 1 \mathrm{H}), 6.03-5.93$ (m, $1 \mathrm{H}), 5.17-5.12(\mathrm{~m}, 1 \mathrm{H}), 5.06-5.03(\mathrm{~m}, 1 \mathrm{H}), 3.64(\mathrm{t}, J=7.3 \mathrm{~Hz}$, $2 \mathrm{H}), 3.32(\mathrm{~s}, 3 \mathrm{H}), 2.62-2.56(\mathrm{~m}, 2 \mathrm{H}), 1.45(\mathrm{~s}, 9 \mathrm{H}) .{ }^{13} \mathrm{C}\left\{{ }^{1} \mathrm{H}\right\}$ NMR (100 MHz, $\left.\mathrm{CDCl}_{3}\right) \delta 171.3(\mathrm{C}), 154.9(\mathrm{C}), 145.1(\mathrm{C})$, $142.0(\mathrm{CH}), 137.0(\mathrm{CH}), 136.2(\mathrm{C}), 126.5(\mathrm{CH}), 126.3(\mathrm{CH})$, $123.6(\mathrm{C}), 115.5\left(\mathrm{CH}_{2}\right), 105.6(\mathrm{CH}), 80.8(\mathrm{C}), 37.9\left(\mathrm{CH}_{3}\right), 36.9$ $\left(\mathrm{CH}_{2}\right), 28.5\left(\mathrm{CH}_{2}\right), 28.3\left(\mathrm{CH}_{3}\right)$. HRMS (ESI-orbitrap) $\mathrm{m} / \mathrm{z}$ : [M $+\mathrm{Na}]^{+}$calcd for $\mathrm{C}_{18} \mathrm{H}_{23} \mathrm{~N}_{3} \mathrm{NaO}_{3}, 352.1632$; found 352.1632 .

1-(3-Methyl-1H-pyrrolo[2,3-b]pyridin-1-yl)pent-4-en-1one (1f): Yield: $111.1 \mathrm{mg}(0.519 \mathrm{mmol}, 74 \%)$ from pent-4enoic acid $(69.9 \mathrm{mg}, 0.698 \mathrm{mmol})$. Colorless oil. IR (KBr) 3128, 2975, 2937, 1699, 1562, 1409, 1386, 1325, 1265, 1218, 1198, 1071, 925, $770 \mathrm{~cm}^{-1} .{ }^{1} \mathrm{H}$ NMR $\left(400 \mathrm{MHz}, \mathrm{CDCl}_{3}\right) \delta$ 8.36 (dd, $J=4.8,1.6 \mathrm{~Hz}, 1 \mathrm{H}), 7.81$ (dd, $J=7.8,1.6 \mathrm{~Hz}, 1 \mathrm{H})$, 7.77 (br q, $J=1.3 \mathrm{~Hz}, 1 \mathrm{H}), 7.20$ (dd, $J=7.8,4.8 \mathrm{~Hz}, 1 \mathrm{H}), 6.04$ $5.93(\mathrm{~m}, 1 \mathrm{H}), 5.17-5.12(\mathrm{~m}, 1 \mathrm{H}), 5.06-5.02(\mathrm{~m}, 1 \mathrm{H}), 3.64(\mathrm{t}$, $J=7.4 \mathrm{~Hz}, 2 \mathrm{H}), 2.63-2.56(\mathrm{~m}, 2 \mathrm{H}), 2.27(\mathrm{~d}, J=1.3 \mathrm{~Hz}, 3 \mathrm{H})$. ${ }^{13} \mathrm{C}\left\{{ }^{1} \mathrm{H}\right\}$ NMR $\left(100 \mathrm{MHz}, \mathrm{CDCl}_{3}\right) \delta 171.1$ (C), 147.9 (C), $143.6(\mathrm{CH}), 137.2(\mathrm{CH}), 127.3(\mathrm{CH}), 124.8(\mathrm{C}), 122.4(\mathrm{CH})$, $118.2(\mathrm{CH}), 115.4\left(\mathrm{CH}_{2}\right), 115.1(\mathrm{C}), 36.9\left(\mathrm{CH}_{2}\right), 28.6\left(\mathrm{CH}_{2}\right)$, $9.7\left(\mathrm{CH}_{3}\right)$. HRMS (ESI-orbitrap) $m / z:[\mathrm{M}+\mathrm{Na}]^{+}$calcd for $\mathrm{C}_{13} \mathrm{H}_{14} \mathrm{~N}_{2} \mathrm{NaO}$, 237.0998; found, 237.1002.

1-(2-Methyl-1H-pyrrolo[2,3-b]pyridin-1-yl)pent-4-en-1one (1g): Yield: $171.0 \mathrm{mg}(0.798 \mathrm{mmol}, 86 \%)$ from pent-4enoic acid $(93.3 \mathrm{mg}, 0.932 \mathrm{mmol})$. Colorless oil. IR (KBr) 3077, 2978, 2925, 1713, 1641, 1592, 1567, 1404, 1288, 1178, 1107, 1025, 913, 988, 811, $767 \mathrm{~cm}^{-1} .{ }^{1} \mathrm{H}$ NMR $(400 \mathrm{MHz}$, $\left.\mathrm{CDCl}_{3}\right) \delta 8.28(\mathrm{dd}, J=4.8,1.6 \mathrm{~Hz}, 1 \mathrm{H}), 7.75(\mathrm{dd}, J=7.8,1.6 \mathrm{~Hz}$, $1 \mathrm{H}), 7.15$ (dd, $J=7.8,4.8 \mathrm{~Hz}, 1 \mathrm{H}), 6.30(\mathrm{br} \mathrm{q}, J=1.0 \mathrm{~Hz}, 1 \mathrm{H})$, 6.03-5.93 (m, 1H), 5.16-5.11 (m, 1H), 5.06-5.02 (m, 1H), $3.72(\mathrm{t}, J=7.3 \mathrm{~Hz}, 2 \mathrm{H}), 2.67(\mathrm{~d}, J=1.0 \mathrm{~Hz}, 3 \mathrm{H}), 2.60-2.55(\mathrm{~m}$, $2 \mathrm{H}) .{ }^{13} \mathrm{C}\left\{{ }^{1} \mathrm{H}\right\}$ NMR $\left(100 \mathrm{MHz}, \mathrm{CDCl}_{3}\right) \delta 173.9(\mathrm{C}), 148.9(\mathrm{C})$, $142.2(\mathrm{CH}), 139.8(\mathrm{C}), 137.3(\mathrm{CH}), 127.3(\mathrm{CH}), 122.0(\mathrm{C})$, $118.5(\mathrm{CH}), 115.3\left(\mathrm{CH}_{2}\right), 105.5(\mathrm{CH}), 38.8\left(\mathrm{CH}_{2}\right), 28.7\left(\mathrm{CH}_{2}\right)$, $17.6\left(\mathrm{CH}_{3}\right)$. HRMS (ESI-orbitrap) $\mathrm{m} / \mathrm{z}:[\mathrm{M}+\mathrm{Na}]^{+}$calcd for $\mathrm{C}_{13} \mathrm{H}_{14} \mathrm{~N}_{2} \mathrm{NaO}$, 237.0998; found, 237.0999.

1-(2,3-Dimethyl-1H-pyrrolo[2,3-b]pyridin-1-yl)pent-4en-1-one (1h): Yield: $132.7 \mathrm{mg}(0.581 \mathrm{mmol}, 78 \%)$ from pent-4-enoic acid $(74.7 \mathrm{mg}, 0.746 \mathrm{mmol})$. Colorless oil. IR (KBr) 3069, 2979, 2925, 1707, 1573, 1420, 1391, 1312, 1278, $1188,1098,912,796,773 \mathrm{~cm}^{-1} .{ }^{1} \mathrm{H}$ NMR (400 MHz, $\left.\mathrm{CDCl}_{3}\right)$ $\delta 8.29$ (dd, $J=4.8,1.6 \mathrm{~Hz}, 1 \mathrm{H}), 7.74(\mathrm{dd}, J=7.7,1.6 \mathrm{~Hz}, 1 \mathrm{H})$, $7.18(\mathrm{dd}, J=7.7,4.8 \mathrm{~Hz}, 1 \mathrm{H}), 6.03-5.92(\mathrm{~m}, 1 \mathrm{H}), 5.15-5.10(\mathrm{~m}$, $1 \mathrm{H}), 5.05-5.01(\mathrm{~m}, 1 \mathrm{H}), 3.71(\mathrm{t}, J=7.3 \mathrm{~Hz}, 2 \mathrm{H}), 2.60(\mathrm{~s}, 3 \mathrm{H})$, 2.60-2.54 (m, 2H), $2.19(\mathrm{~d}, J=0.8 \mathrm{~Hz}, 3 \mathrm{H}) .{ }^{13} \mathrm{C}\left\{{ }^{1} \mathrm{H}\right\}$ NMR $\left(100 \mathrm{MHz}, \mathrm{CDCl}_{3}\right) \delta 173.8(\mathrm{C}), 148.4(\mathrm{C}), 142.2(\mathrm{CH}), 137.5$ $(\mathrm{CH}), 135.0(\mathrm{C}), 125.7(\mathrm{CH}), 123.5(\mathrm{C}), 118.2(\mathrm{CH}), 115.2$ $\left(\mathrm{CH}_{2}\right), 111.9(\mathrm{C}), 38.8\left(\mathrm{CH}_{2}\right), 28.9\left(\mathrm{CH}_{2}\right), 14.1\left(\mathrm{CH}_{3}\right), 8.2$ $\left(\mathrm{CH}_{3}\right)$. HRMS (ESI-orbitrap) $\mathrm{m} / \mathrm{z}:[\mathrm{M}+\mathrm{Na}]^{+}$calcd for $\mathrm{C}_{14} \mathrm{H}_{16} \mathrm{~N}_{2} \mathrm{NaO}$, 251.1155; found, 251.1154.

1-(1H-Pyrrolo[2,3-b]pyridin-1-yl)hexa-4,5-dien-1-one (1i): Yield: $208.6 \mathrm{mg}(0.983 \mathrm{mmol}, 82 \%)$ from hexa-4,5dienoic acid $\left(0.83 \mathrm{~g}\right.$ of approx. $16 \%(\mathrm{w} / \mathrm{w})$ solution in $\mathrm{Et}_{2} \mathrm{O}$, $1.2 \mathrm{mmol}$ ). Faintly yellow oil. IR (KBr) 3154, 3117, 2943, 2922, 1956, 1709, 1579, 1532, 1409, 1382, 1300, 1262, 1202, 860, 806, $741 \mathrm{~cm}^{-1} .{ }^{1} \mathrm{H}$ NMR $\left(400 \mathrm{MHz}, \mathrm{CDCl}_{3}\right) \delta 8.37$ (dd, $J=4.8,1.6 \mathrm{~Hz}, 1 \mathrm{H}), 8.00(\mathrm{~d}, J=4.1 \mathrm{~Hz}, 1 \mathrm{H}), 7.88$ (dd, $J=7.8$, $1.6 \mathrm{~Hz}, 1 \mathrm{H}), 7.20$ (dd, J=7.8, $4.8 \mathrm{~Hz}, 1 \mathrm{H}), 6.60$ (d, $J=4.1 \mathrm{~Hz}$, $1 \mathrm{H}), 5.34-5.27(\mathrm{~m}, 1 \mathrm{H}), 4.71-4.68(\mathrm{~m}, 2 \mathrm{H}), 3.70(\mathrm{t}, J=7.2 \mathrm{~Hz}$, 2H), 2.59-2.52 (m, 2H). ${ }^{13} \mathrm{C}\left\{{ }^{1} \mathrm{H}\right\}$ NMR $\left(100 \mathrm{MHz}, \mathrm{CDCl}_{3}\right) \delta$
208.5 (C), 171.4 (C), $147.7(\mathrm{C}), 143.8(\mathrm{CH}), 129.3(\mathrm{CH})$, $125.5(\mathrm{CH}), 123.8(\mathrm{C}), 118.6(\mathrm{CH}), 105.7(\mathrm{CH}), 89.0(\mathrm{CH})$, $75.7\left(\mathrm{CH}_{2}\right), 37.1\left(\mathrm{CH}_{2}\right), 23.1\left(\mathrm{CH}_{2}\right)$. HRMS (ESI-orbitrap) $m / z:[\mathrm{M}+\mathrm{H}]^{+}$calcd for $\mathrm{C}_{13} \mathrm{H}_{12} \mathrm{~N}_{2} \mathrm{NaO}, 235.0842$; found, 235.0843 .

5-Methyl-1-(1H-pyrrolo[2,3-b]pyridin-1-yl)hex-4-en-1one (1j): Yield: $162.0 \mathrm{mg}(0.710 \mathrm{mmol}, 71 \%)$ from 5methylhex-4-enoic acid (128.7 mg, $1.00 \mathrm{mmol})$. Colorless oil. IR (KBr) 3148, 3050, 2968, 2913, 1709, 1579, 1530, 1407, $1302,1262,1202,1102,1064,985,890,803,777,732 \mathrm{~cm}^{-1}$. ${ }^{1} \mathrm{H}$ NMR (400 MHz, $\left.\mathrm{CDCl}_{3}\right) \delta 8.37(\mathrm{dd}, J=4.8,1.6 \mathrm{~Hz}, 1 \mathrm{H})$, $8.01(\mathrm{~d}, J=4.1 \mathrm{~Hz}, 1 \mathrm{H}), 7.88$ (dd, $J=7.8,1.6 \mathrm{~Hz}, 1 \mathrm{H}), 7.19$ (dd, $J=7.8,4.8 \mathrm{~Hz}, 1 \mathrm{H}), 6.59$ (d, $J=4.1 \mathrm{~Hz}, 1 \mathrm{H}), 5.30-5.25$ (m, 1H), $3.58(\mathrm{t}, J=7.4 \mathrm{~Hz}, 2 \mathrm{H}), 2.56-2.50(\mathrm{~m}, 2 \mathrm{H}), 1.71(\mathrm{~d}, J=1.0 \mathrm{~Hz}$, $3 \mathrm{H}), 1.66(\mathrm{~s}, 3 \mathrm{H}) .{ }^{13} \mathrm{C}\left\{{ }^{1} \mathrm{H}\right\}$ NMR $\left(100 \mathrm{MHz}, \mathrm{CDCl}_{3}\right) \delta 171.9$ (C), $147.6(\mathrm{C}), 143.7(\mathrm{CH}), 132.9(\mathrm{C}), 129.2(\mathrm{CH}), 125.5(\mathrm{CH})$, $123.7(\mathrm{C}), 122.8(\mathrm{CH}), 118.6(\mathrm{CH}), 105.6(\mathrm{CH}), 37.9\left(\mathrm{CH}_{2}\right)$, $25.7\left(\mathrm{CH}_{3}\right), 23.3\left(\mathrm{CH}_{2}\right), 17.7\left(\mathrm{CH}_{3}\right)$. HRMS (ESI-orbitrap) $m / z:\left(\mathrm{M}+\mathrm{Na}^{+}\right)$calcd for $\mathrm{C}_{14} \mathrm{H}_{16} \mathrm{~N}_{2} \mathrm{NaO}, 251.1155$; found, 251.1158

2-(Cyclohex-2-en-1-yl)-1-(1H-pyrrolo[2,3-b]pyridin-1yl)ethan-1-one (1k): Yield: $186.8 \mathrm{mg}(0.777 \mathrm{mmol}, 69 \%)$ from 2-(cyclohex-2-en-1-yl)acetic acid (156.8 mg, $1.12 \mathrm{mmol}$ ). Colorless oil. IR (KBr) 3147, 3019, 2926, 1710, 1579, 1530, $1408,1301,1263,1198,1106,1050,890,803,778,735 \mathrm{~cm}^{-1}$. ${ }^{1} \mathrm{H}$ NMR $\left(400 \mathrm{MHz}, \mathrm{CDCl}_{3}\right) \delta 8.37(\mathrm{dd}, J=4.8,1.6 \mathrm{~Hz}, 1 \mathrm{H})$, $8.01(\mathrm{~d}, J=4.1 \mathrm{~Hz}, 1 \mathrm{H}), 7.88(\mathrm{dd}, J=7.8,1.6 \mathrm{~Hz}, 1 \mathrm{H}), 7.19$ (dd, $J=7.8,4.8 \mathrm{~Hz}, 1 \mathrm{H}), 6.59(\mathrm{~d}, J=4.1 \mathrm{~Hz}, 1 \mathrm{H}), 5.78-5.71(\mathrm{~m}, 2 \mathrm{H})$, 3.58-3.56 (m, 2H), 2.91-2.84 (m, 1H), 2.04-1.99 (m, 2H), $1.98-1.91(\mathrm{~m}, 1 \mathrm{H}), 1.81-1.73(\mathrm{~m}, 1 \mathrm{H}), 1.64-1.54(\mathrm{~m}, 1 \mathrm{H})$, $1.50-1.42(\mathrm{~m}, 1 \mathrm{H}) .{ }^{13} \mathrm{C}\left\{{ }^{1} \mathrm{H}\right\} \mathrm{NMR}\left(100 \mathrm{MHz}, \mathrm{CDCl}_{3}\right) \delta 171.2$ (C), $147.6(\mathrm{C}), 143.8(\mathrm{CH}), 130.7(\mathrm{CH}), 129.3(\mathrm{CH}), 128.0$ $(\mathrm{CH}), 125.6(\mathrm{CH}), 123.8(\mathrm{C}), 118.6(\mathrm{CH}), 105.6(\mathrm{CH}), 43.7$ $\left(\mathrm{CH}_{2}\right), 31.9(\mathrm{CH}), 28.8\left(\mathrm{CH}_{2}\right), 25.1\left(\mathrm{CH}_{2}\right), 21.1\left(\mathrm{CH}_{2}\right)$. HRMS (ESI-orbitrap) $m / z:[\mathrm{M}+\mathrm{Na}]^{+}$calcd for $\mathrm{C}_{15} \mathrm{H}_{16} \mathrm{~N}_{2} \mathrm{NaO}$, 263.1155; found, 263.1155.

Diethyl 2-allyl-2-((1-(methoxycarbonyl)-1H-pyrrolo[2,3b]pyridin-3-yl)methyl)malonate (7): This compound was synthesized by a procedure analogous to that for the corresponding indole derivative described in the literature. ${ }^{12 \mathrm{~b}}$ Yield: $23.0 \mathrm{mg} \quad(0.0592 \mathrm{mmol}, 26 \%)$ from diethyl 2-((1(methoxycarbonyl)-1H-pyrrolo[2,3-b]pyridin-3-

yl)methyl)malonate (78.1 mg, $0.224 \mathrm{mmol})$. Colorless oil. IR (KBr) 2981, 1733, 1558, 1442, 1411, 1357, 1325, 1245, 1211, 1133, 1095, 1045, $770 \mathrm{~cm}^{-1} .{ }^{1} \mathrm{H}$ NMR (400 MHz, $\left.\mathrm{CDCl}_{3}\right) \delta$ 8.47 (dd, $J=4.8,1.4 \mathrm{~Hz}, 1 \mathrm{H}), 7.84(\mathrm{dd}, J=7.9,1.4 \mathrm{~Hz}, 1 \mathrm{H})$, 7.56 (s, 1H), 7.19 (dd, $J=7.9,4.8 \mathrm{~Hz}, 1 \mathrm{H}), 5.80-5.69(\mathrm{~m}, 1 \mathrm{H})$, $5.17-5.10(\mathrm{~m}, 2 \mathrm{H}), 4.17-4.03(\mathrm{~m}, 7 \mathrm{H}$, including $\mathrm{s}, 3 \mathrm{H}$ at 4.08$)$, $3.28(\mathrm{~s}, 2 \mathrm{H}), 2.67(\mathrm{~d}, J=7.2 \mathrm{~Hz}, 2 \mathrm{H}), 1.16(\mathrm{t}, J=7.2 \mathrm{~Hz}, 6 \mathrm{H})$. ${ }^{13} \mathrm{C}\left\{{ }^{1} \mathrm{H}\right\}$ NMR $\left(100 \mathrm{MHz}, \mathrm{CDCl}_{3}\right) \delta 170.7(\mathrm{C}), 150.3(\mathrm{C})$, $147.2(\mathrm{C}), 145.0(\mathrm{CH}), 132.2(\mathrm{CH}), 127.9(\mathrm{CH}), 125.3(\mathrm{CH})$, $123.9(\mathrm{C}), 119.6\left(\mathrm{CH}_{2}\right), 118.4(\mathrm{CH}), 113.3(\mathrm{C}), 61.5\left(\mathrm{CH}_{2}\right)$, 58.0 (C), $54.3\left(\mathrm{CH}_{3}\right), 37.4\left(\mathrm{CH}_{2}\right), 27.6\left(\mathrm{CH}_{2}\right), 13.9\left(\mathrm{CH}_{3}\right)$. HRMS (ESI-orbitrap) $m / z[\mathrm{M}+\mathrm{Na}]^{+}$calcd for $\mathrm{C}_{20} \mathrm{H}_{24} \mathrm{~N}_{2} \mathrm{NaO}_{6}$, 411.1527; found, 411.1524 .

General procedure for the photocycloaddition and physical data of the products. The reaction of $1 \mathrm{a}$ is representative.

A small scale reaction for screening of Lewis acids (Table 1, Entry 2). In a Pyrex test tube, 1a (21.2 mg, 0.106 mmol), 3',4'-dimethoxyacetophenone (3) (9.5 mg, 0.053 $\mathrm{mmol})$, and $\mathrm{Mg}(\mathrm{OTf})_{2}(17.9 \mathrm{mg}, 0.0555 \mathrm{mmol})$ were charged. Air present in the tube was replaced with argon by several 
rapid evacuations/Ar introduction cycles. Ethyl acetate (11 $\mathrm{mL}$ ) that had been degassed by three freeze-thaw cycles was transferred into the tube by a syringe. The solution was homogenized by sonication for several seconds, then externally irradiated by a $100 \mathrm{~W}$ high-pressure mercury lamp (UVL100HA; RIKO) for $3 \mathrm{~h}$. The reaction mixture was transferred to a separatory funnel and washed with $1 \mathrm{~mol} / \mathrm{L}$ aqueous solution of sodium hydroxide. The aqueous phase was extracted with dichloromethane two times, and the combined organic phase was dried over anhydrous sodium sulfate. After the removal of sodium sulfate by filtration, the solution was evaporated and triphenylmethane $(12.4 \mathrm{mg}, 0.0508 \mathrm{mmol})$ was added to the mixture as an internal standard. The yield of $\mathbf{2 a}$ and the recovery of $1 \mathrm{a}$ were estimated by ${ }^{1} \mathrm{H}$ NMR integrals.

A preparative scale reaction (Table 2, Entry 3). In a Pyrex $100 \mathrm{~mL}$ photoreactor (UVL-100HA-50P-type; RIKO), 3',4'-dimethoxyacetophenone (3) (86.8 $\mathrm{mg}, 0.482 \mathrm{mmol})$ and $\mathrm{Mg}(\mathrm{OTf})_{2}(307.8 \mathrm{mg}, 0.955 \mathrm{mmol}$ ) were charged, and air present in the vessel was replaced with argon by several rapid evacuations/Ar introduction cycles. Compound 1a (190.3 mg, $0.950 \mathrm{mmol}$ ) dissolved in ethyl acetate that had been degassed by passing nitrogen through it for $1 \mathrm{~h}$ was transferred to the vessel by a Teflon cannula, followed by washing with the ethyl acetate several times. The total amount of the solvent was $95 \mathrm{~mL}$. The solution was irradiated by a $100 \mathrm{~W}$ highpressure mercury lamp internally for $2 \mathrm{~h}$. The reaction mixture was transferred to a separatory funnel and washed with 1 $\mathrm{mol} / \mathrm{L}$ aqueous solution of sodium hydroxide. The aqueous phase was extracted with dichloromethane two times, and the combined organic phase was dried over anhydrous sodium sulfate. After the removal of sodium sulfate by filtration, the solution was concentrated under reduced pressure. The crude material was purified by silica-gel column chromatography to give the product $2 \mathrm{a}(169.5 \mathrm{mg}, 0.846 \mathrm{mmol}, 89 \%)$ as a colorless solid.

rac-(1a $R, 9 \mathrm{~b} R, 9 \mathrm{c} R)-1,1 \mathrm{a},, 2,3,9 \mathrm{~b}, 9 \mathrm{c}-H e x a h y d r o-4 H-$ cyclobuta $[\boldsymbol{h i}]$ pyrido[3,2-b]indolizin-4-one (2a): Colorless solid. IR (KBr) 3047, 2984, 2950, 1686, 1599, 1412, 1379, 1336, 1302, 1220, 1144, 800, $781 \mathrm{~cm}^{-1}$. ${ }^{1} \mathrm{H}$ NMR $(400 \mathrm{MHz}$, $\left.\mathrm{CDCl}_{3}\right) \delta 8.26(\mathrm{dd}, J=5.3,1.7 \mathrm{~Hz}, 1 \mathrm{H}), 7.36(\mathrm{ddd}, J=7.3,1.7$, $0.7 \mathrm{~Hz}, 1 \mathrm{H}), 6.88(\mathrm{dd}, J=7.3,5.3 \mathrm{~Hz}, 1 \mathrm{H}), 4.66\left(\mathrm{dt}, J_{\mathrm{d}}=2.9\right.$, $\left.J_{\mathrm{t}}=6.2 \mathrm{~Hz}, 1 \mathrm{H}\right), 3.65-3.60(\mathrm{~m}, 1 \mathrm{H}), 3.15-3.05(\mathrm{~m}, 1 \mathrm{H}), 2.89$ $2.81(\mathrm{~m}, 1 \mathrm{H}), 2.59-2.53(\mathrm{~m}, 1 \mathrm{H}), 2.31-2.16(\mathrm{~m}, 2 \mathrm{H}), 1.98$ (ddd, $J=12.2,8.8,7.4 \mathrm{~Hz}, 1 \mathrm{H}), 1.66-1.55(\mathrm{~m}, 1 \mathrm{H}) .{ }^{13} \mathrm{C}\left\{{ }^{1} \mathrm{H}\right\}$ NMR (100 MHz, $\left.\mathrm{CDCl}_{3}\right) \delta 170.7(\mathrm{C}), 159.2(\mathrm{C}), 146.7(\mathrm{CH})$, $131.7(\mathrm{CH}), 129.5(\mathrm{C}), 118.8(\mathrm{CH}), 61.7(\mathrm{CH}), 36.8\left(\mathrm{CH}_{2}\right)$, $35.6\left(\mathrm{CH}_{2}\right), 35.4(\mathrm{CH}), 30.4(\mathrm{CH}), 26.5\left(\mathrm{CH}_{2}\right)$. HRMS (ESIorbitrap) $m / z$ : $[\mathrm{M}+\mathrm{Na}]^{+}$calcd for $\mathrm{C}_{12} \mathrm{H}_{12} \mathrm{~N}_{2} \mathrm{NaO}, 223.0842$; found, 223.0843. Anal. Calcd for $\mathrm{C}_{12} \mathrm{H}_{12} \mathrm{~N}_{2} \mathrm{O}: \mathrm{C}, 71.98 ; \mathrm{H}$, 6.04; N, 13.99. Found: C, 71.80; H, 6.01; N, 13.89.

Physical data of the other products were as follows:

rac-(1a $R, 9 \mathrm{~b} R, 9 \mathrm{c} R)-9-C h l o r o-1,1 \mathrm{a}, 2,3,9 \mathrm{~b}, 9 \mathrm{c}-h$ exahydro4H-cyclobuta $[h i]$ pyrido[3,2-b]indolizin-4-one (2b): Yield: $33.5 \mathrm{mg}(0.143 \mathrm{mmol}, 60 \%)$ from $1 \mathbf{b}(56.1 \mathrm{mg}, 0.239 \mathrm{mmol})$. Colorless solid. IR (KBr) 3055, 2963, 1693, 1590, 1562, 1389 , 1336, 1300, 1207, 1138, 1004, 843, $795 \mathrm{~cm}^{-1}$. ${ }^{1} \mathrm{H}$ NMR $(400$ $\left.\mathrm{MHz}, \mathrm{CDCl}_{3}\right) \delta 8.17(\mathrm{~d}, J=5.5 \mathrm{~Hz}, 1 \mathrm{H}), 6.91(\mathrm{~d}, J=5.5 \mathrm{~Hz}, 1 \mathrm{H})$, $4.69\left(\mathrm{dt}, J_{\mathrm{d}}=2.8, J_{\mathrm{t}}=6.4 \mathrm{~Hz}, 1 \mathrm{H}\right), 3.79(\mathrm{q}, J=7.3 \mathrm{~Hz}, 1 \mathrm{H}), 3.21-$ $3.11(\mathrm{~m}, 1 \mathrm{H}), 2.90-2.98(\mathrm{~m}, 1 \mathrm{H}), 2.58\left(\mathrm{dt}, J_{\mathrm{d}}=14.2, J_{\mathrm{t}}=3.2 \mathrm{~Hz}\right.$, $1 \mathrm{H}), 2.35-2.17(\mathrm{~m}, 2 \mathrm{H}), 2.05-1.98(\mathrm{~m}, 1 \mathrm{H}), 1.68-1.59(\mathrm{~m}$, 1H). ${ }^{13} \mathrm{C}\left\{{ }^{1} \mathrm{H}\right\}$ NMR $\left(100 \mathrm{MHz}, \mathrm{CDCl}_{3}\right) \delta 170.6(\mathrm{C}), 159.9(\mathrm{C})$, $147.8(\mathrm{CH}), 139.1(\mathrm{C}), 128.3(\mathrm{C}), 119.3(\mathrm{CH}), 61.3(\mathrm{CH}), 35.8$
$\left(\mathrm{CH}_{2}\right), 35.5\left(\mathrm{CH}_{2}\right), 34.4(\mathrm{CH}), 30.4(\mathrm{CH}), 26.3\left(\mathrm{CH}_{2}\right)$. HRMS (ESI-orbitrap) $m / z$ : $[\mathrm{M}+\mathrm{Na}]^{+}$calcd for $\mathrm{C}_{12} \mathrm{H}_{11}{ }^{35} \mathrm{ClN}_{2} \mathrm{NaO}$, 257.0452; found, 257.0454.

rac-Methyl (1aR,9bR,9cR)-1a,2,3,4,9b,9c-hexahydro-4oxo-1 $H$-cyclobuta $[h i]$ pyrido $[3,2-b]$ indolizine-8-carboxylate (2c): Yield: $35.9 \mathrm{mg}(0.139 \mathrm{mmol}, 64 \%)$ from $1 \mathrm{c}(56.1 \mathrm{mg}$, $0.217 \mathrm{mmol}$ ). Colorless solid. IR (KBr) 2952, 1716, 1693, $1609,1441,1396,1334,1308,1270,1234,1201,1094,984$, $786 \mathrm{~cm}^{-1}$. ${ }^{1} \mathrm{H}$ NMR $\left(400 \mathrm{MHz}, \mathrm{CDCl}_{3}\right) \delta 8.91(\mathrm{~d}, J=2.1 \mathrm{~Hz}$, $1 \mathrm{H}), 7.95(\mathrm{dd}, J=2.1,0.7 \mathrm{~Hz}, 1 \mathrm{H}), 4.72\left(\mathrm{dt}, J_{\mathrm{d}}=2.9, J_{\mathrm{t}}=6.2 \mathrm{~Hz}\right.$, $1 \mathrm{H}), 3.90(\mathrm{~s}, 3 \mathrm{H}), 3.73-3.67(\mathrm{~m}, 1 \mathrm{H}), 3.20-3.10(\mathrm{~m}, 1 \mathrm{H})$, 2.94-2.86 (m, 1H), 2.63-2.57 (m, 1H), 2.35-2.20 (m, 2H), 2.00 (ddd, $J=12.4,8.7,7.5 \mathrm{~Hz}, 1 \mathrm{H}), 1.68-1.58(\mathrm{~m}, 1 \mathrm{H})$. ${ }^{13} \mathrm{C}\left\{{ }^{1} \mathrm{H}\right\}$ NMR $\left(100 \mathrm{MHz}, \mathrm{CDCl}_{3}\right) \delta 170.6(\mathrm{C}), 165.6(\mathrm{C})$, $162.0(\mathrm{C}), 149.6(\mathrm{CH}), 132.3(\mathrm{CH}), 129.5(\mathrm{C}), 121.4(\mathrm{C}), 62.0$ $(\mathrm{CH}), 52.1\left(\mathrm{CH}_{3}\right), 36.7\left(\mathrm{CH}_{2}\right), 35.6\left(\mathrm{CH}_{2}\right), 34.8(\mathrm{CH}), 30.5$ $(\mathrm{CH}), 26.2\left(\mathrm{CH}_{2}\right)$. HRMS (ESI-orbitrap) $\mathrm{m} / \mathrm{z}$ : $[\mathrm{M}+\mathrm{Na}]^{+}$calcd for $\mathrm{C}_{14} \mathrm{H}_{14} \mathrm{~N}_{2} \mathrm{NaO}_{3}, 281.0897$; found, 281.0899 .

rac-(1aR,9bR,9cR)-1,1a,,2,3,9b,9c-Hexahydro-9methoxy-4H-cyclobuta[ $[h i]$ pyrido $[3,2-b]$ indolizin-4-one (2d): Yield: $48.7 \mathrm{mg}$ (containing a small amount of AcOEt, $0.205 \mathrm{mmol}$ estimated by ${ }^{1} \mathrm{H}$ NMR, $\left.88 \%\right)$ from $1 d(53.4 \mathrm{mg}$, $0.232 \mathrm{mmol}$ ). Faintly yellow solid. IR (KBr) 3034, 2975, 2939, $1692,1598,1575,1397,1365,1283,1206,1186,1080,822$ $\mathrm{cm}^{-1} .{ }^{1} \mathrm{H}$ NMR (400 MHz, $\left.\mathrm{CDCl}_{3}\right) \delta 8.20(\mathrm{~d}, J=6.0 \mathrm{~Hz}, 1 \mathrm{H})$, $6.52(\mathrm{~d}, J=6.0 \mathrm{~Hz}, 1 \mathrm{H}), 4.64\left(\mathrm{dt}, J_{\mathrm{d}}=2.9, J_{\mathrm{t}}=6.4 \mathrm{~Hz}, 1 \mathrm{H}\right), 3.84$ $(\mathrm{s}, 3 \mathrm{H}), 3.73-3.67(\mathrm{~m}, 1 \mathrm{H}), 3.14-3.04(\mathrm{~m}, 1 \mathrm{H}), 2.91-2.82(\mathrm{~m}$, $1 \mathrm{H}), 2.57-2.52(\mathrm{~m}, 1 \mathrm{H}), 2.31-2.14(\mathrm{~m}, 2 \mathrm{H}), 1.95$ (ddd, $J=12.2$, 8.7, $7.4 \mathrm{~Hz}, 1 \mathrm{H}), 1.66-1.57(\mathrm{~m}, 1 \mathrm{H}) .{ }^{13} \mathrm{C}\left\{{ }^{1} \mathrm{H}\right\} \mathrm{NMR}(100 \mathrm{MHz}$, $\left.\mathrm{CDCl}_{3}\right) \delta 170.6(\mathrm{C}), 161.5(\mathrm{C}), 160.8(\mathrm{C}), 149.0(\mathrm{CH}), 115.5$ (C), $103.6(\mathrm{CH}), 61.9(\mathrm{CH}), 55.3\left(\mathrm{CH}_{3}\right), 36.4\left(\mathrm{CH}_{2}\right), 35.5$ $\left(\mathrm{CH}_{2}\right), 32.5(\mathrm{CH}), 30.2(\mathrm{CH}), 26.4\left(\mathrm{CH}_{2}\right)$. HRMS (ESIorbitrap) $m / z:[\mathrm{M}+\mathrm{Na}]^{+}$calcd for $\mathrm{C}_{13} \mathrm{H}_{14} \mathrm{~N}_{2} \mathrm{NaO}_{2}, 253.0948$; found, 253.0950.

rac-tert-Butyl $N$-methyl- $N$-((1a $R, 9 \mathrm{~b} R, 9 \mathrm{c} R)-1 \mathrm{a}, 2,3,4,9 \mathrm{~b}, 9 \mathrm{c}-$ hexahydro-4-oxo- $1 H$-cyclobuta $[h i]$ pyrido $[3,2-b]$ indolizin8-yl)carbamate (2e): Yield: $10.8 \mathrm{mg}$ (containing a small amount of AcOEt, $0.0318 \mathrm{mmol}$ estimated by ${ }^{1} \mathrm{H}$ NMR, 77\%) from 1e (13.6 mg, $0.0413 \mathrm{mmol})$. Colorless oil. IR (KBr) 2976, 2934, 1694, 1469, 1404, 1366, 1353, 1332, 1222, 1151, 776, $731 \mathrm{~cm}^{-1} .{ }^{1} \mathrm{H}$ NMR $\left(400 \mathrm{MHz}, \mathrm{CDCl}_{3}\right) \delta 8.10(\mathrm{~d}, J=2.4 \mathrm{~Hz}$, $1 \mathrm{H}), 1.42(\mathrm{~s}, 9 \mathrm{H}), 7.35($ br s, $1 \mathrm{H}), 4.69\left(\mathrm{dt}, J_{\mathrm{d}}=2.9, J_{\mathrm{t}}=6.2 \mathrm{~Hz}\right.$, $1 \mathrm{H}), 3.62$ (q, $J=7.3 \mathrm{~Hz}, 1 \mathrm{H}), 3.22(\mathrm{~s}, 3 \mathrm{H}), 3.15-3.04(\mathrm{~m}, 1 \mathrm{H})$, $2.90-2.81(\mathrm{~m}, 1 \mathrm{H}), 2.57-2.53(\mathrm{~m}, 1 \mathrm{H}), 2.32-2.16(\mathrm{~m}, 2 \mathrm{H})$, 1.98 (ddd, $J=12.3,8.7,7.6 \mathrm{~Hz}, 1 \mathrm{H}), 1.66-1.57(\mathrm{~m}, 1 \mathrm{H})$. ${ }^{13} \mathrm{C}\left\{{ }^{1} \mathrm{H}\right\}$ NMR $\left(100 \mathrm{MHz}, \mathrm{CDCl}_{3}\right) \delta 170.5(\mathrm{C}), 156.1(\mathrm{C})$, 154.5 (C), 142.6 (br, CH), 136.4 (C), $130.1(\mathrm{CH}), 129.7$ (C), $80.9(\mathrm{C}), 62.4(\mathrm{CH}), 37.4\left(\mathrm{CH}_{3}\right), 36.7\left(\mathrm{CH}_{2}\right), 35.5\left(\mathrm{CH}_{2}\right), 35.2$ $(\mathrm{CH}), 30.4(\mathrm{CH}), 28.2\left(\mathrm{CH}_{3}\right), 26.5\left(\mathrm{CH}_{2}\right)$. HRMS (ESIorbitrap) $m / z:[\mathrm{M}+\mathrm{Na}]^{+}$calcd for $\mathrm{C}_{18} \mathrm{H}_{23} \mathrm{~N}_{3} \mathrm{NaO}_{3}, 352.1632$; found, 352.1632 .

rac-(1a $R, 9 \mathrm{~b} R, 9 \mathrm{c} R)-1,1 \mathrm{a}, 2,3,9 \mathrm{~b}, 9 \mathrm{c}-H e x a h y d r o-9 \mathrm{~b}-m e t h y l-$ 4H-cyclobuta $[\boldsymbol{h i}]$ pyrido[3,2-b]indolizin-4-one (2f): Yield: $43.7 \mathrm{mg}$ (0.204 mmol, 93\%) from 1f (47.1 mg, $0.220 \mathrm{mmol})$. Pale yellow oil. IR (KBr) 3048, 2954, 2927, 1688, 1597, 1412, 1368, 1319, 1259, 1151, 1116, $781 \mathrm{~cm}^{-1} .{ }^{1} \mathrm{H}$ NMR $(400 \mathrm{MHz}$, $\left.\mathrm{CDCl}_{3}\right) \delta 8.29(\mathrm{dd}, J=5.3,1.6 \mathrm{~Hz}, 1 \mathrm{H}), 7.36(\mathrm{dd}, J=7.3,1.6 \mathrm{~Hz}$, $1 \mathrm{H}), 6.95$ (dd, $J=7.3,5.3 \mathrm{~Hz}, 1 \mathrm{H}), 4.29$ (dd, $J=6.1,3.2 \mathrm{~Hz}, 1 \mathrm{H})$, 3.16-3.06 (m, 1H), 2.59-2.55 (m, 1H), 2.46 (ddd, $J=12.0,9.1$, $3.2 \mathrm{~Hz}, 1 \mathrm{H}), 2.35-2.19(\mathrm{~m}, 2 \mathrm{H}), 2.09(\mathrm{dd}, J=12.0,8.9 \mathrm{~Hz}, 1 \mathrm{H})$, 1.66-1.60 (m, 1H), $1.58(\mathrm{~s}, 3 \mathrm{H}) .{ }^{13} \mathrm{C}\left\{{ }^{1} \mathrm{H}\right\}$ NMR (100 MHz, $\left.\mathrm{CDCl}_{3}\right) \delta 170.5(\mathrm{C}), 158.4(\mathrm{C}), 146.5(\mathrm{CH}), 133.2(\mathrm{C}), 129.8$ 
$(\mathrm{CH}), 118.8(\mathrm{CH}), 65.9(\mathrm{CH}), 42.3\left(\mathrm{CH}_{2}\right), 41.6(\mathrm{C}), 35.5$ $\left(\mathrm{CH}_{2}\right), 27.5(\mathrm{CH}), 25.7\left(\mathrm{CH}_{2}\right), 20.0\left(\mathrm{CH}_{3}\right)$. HRMS (ESIorbitrap) $m / z$ : $[\mathrm{M}+\mathrm{Na}]^{+}$calcd for $\mathrm{C}_{13} \mathrm{H}_{14} \mathrm{~N}_{2} \mathrm{NaO}, 237.0998$; found, 237.1000 .

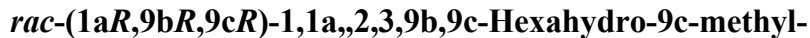
4H-cyclobuta $[h i]$ pyrido $[3,2-b]$ indolizin-4-one (2g): Yield: $31.1 \mathrm{mg}(0.145 \mathrm{mmol}, 62 \%)$ from $1 \mathrm{~g}(50.3 \mathrm{mg}, 0.235 \mathrm{mmol})$. Pale yellow oil. IR (KBr) 3048, 2957, 2866, 1688, 1596, 1410, $1370,1313,1301,1216,1157,1050,781 \mathrm{~cm}^{-1} .{ }^{1} \mathrm{H}$ NMR $(400$ $\left.\mathrm{MHz}, \mathrm{CDCl}_{3}\right) \delta 8.29(\mathrm{dd}, J=5.3,1.6 \mathrm{~Hz}, 1 \mathrm{H}), 7.40(\mathrm{ddd}, J=7.3$, $1.6,0.5 \mathrm{~Hz}, 1 \mathrm{H}), 6.92(\mathrm{dd}, J=7.3,5.3 \mathrm{~Hz}, 1 \mathrm{H}), 3.21(\mathrm{t}, J=8.2$ $\mathrm{Hz}, 1 \mathrm{H}), 2.86-2.73(\mathrm{~m}, 2 \mathrm{H}), 2.56-2.50(\mathrm{~m}, 1 \mathrm{H}), 2.42-2.28(\mathrm{~m}$, $2 \mathrm{H}), 1.77-1.59(\mathrm{~m}, 2 \mathrm{H}), 1.47(\mathrm{~s}, 3 \mathrm{H}) .{ }^{13} \mathrm{C}\left\{{ }^{1} \mathrm{H}\right\}$ NMR $(100$ $\left.\mathrm{MHz}, \mathrm{CDCl}_{3}\right) \delta 170.1(\mathrm{C}), 158.2(\mathrm{C}), 146.6(\mathrm{CH}), 132.2(\mathrm{CH})$, $128.5(\mathrm{C}), 119.0(\mathrm{CH}), 68.9(\mathrm{CH}), 40.8(\mathrm{CH}), 35.8(\mathrm{CH}), 33.6$ $\left(\mathrm{CH}_{2}\right), 33.5\left(\mathrm{CH}_{2}\right), 27.1\left(\mathrm{CH}_{2}\right), 25.8\left(\mathrm{CH}_{3}\right)$. HRMS (ESIorbitrap) $m / z:[\mathrm{M}+\mathrm{Na}]^{+}$calcd for $\mathrm{C}_{13} \mathrm{H}_{14} \mathrm{~N}_{2} \mathrm{NaO}, 237.0998$; found, 237.1002 .

rac-(1a $R, 9 \mathrm{~b} R, 9 \mathrm{c} R)-1,1 \mathrm{a}, 2,3,9 \mathrm{~b}, 9 \mathrm{c}-H e x a h y d r o-9 \mathrm{~b}, 9 \mathrm{c}-$ dimethyl-4H-cyclobuta[hi]pyrido[3,2-b]indolizin-4-one (2h): Yield: $13.8 \mathrm{mg}(0.0604 \mathrm{mmol}, 28 \%)$ from $\mathbf{1 h}(49.4 \mathrm{mg}$, $0.216 \mathrm{mmol}$ ). Colorless solid. IR (KBr) 3049, 2963, 2931, $2868,1685,1596,1412,1369,1315,1301,1146,1120,781$ $\mathrm{cm}^{-1} .{ }^{1} \mathrm{H}$ NMR $\left(400 \mathrm{MHz}, \mathrm{CDCl}_{3}\right) \delta 8.30(\mathrm{dd}, J=5.3,1.7 \mathrm{~Hz}$, 1H), 7.34 (dd, $J=7.3,1.7 \mathrm{~Hz}, 1 \mathrm{H}), 6.96$ (dd, $J=7.3,5.3 \mathrm{~Hz}, 1 \mathrm{H})$, 2.87-2.79 (m, 1H), 2.56 (ddd, $J=14.5,4.5,2.3 \mathrm{~Hz}, 1 \mathrm{H}), 2.47-$ $2.30(\mathrm{~m}, 3 \mathrm{H}), 1.94(\mathrm{dd}, J=11.9,8.9 \mathrm{~Hz}, 1 \mathrm{H}), 1.71-1.61(\mathrm{~m}$, $1 \mathrm{H}), 1.45(\mathrm{~s}, 3 \mathrm{H}), 1.35(\mathrm{~s}, 3 \mathrm{H}) .{ }^{13} \mathrm{C}\left\{{ }^{1} \mathrm{H}\right\}$ NMR $(100 \mathrm{MHz}$, $\left.\mathrm{CDCl}_{3}\right) \delta 170.1(\mathrm{C}), 157.8(\mathrm{C}), 146.8(\mathrm{CH}), 132.6(\mathrm{C}), 130.4$ $(\mathrm{CH}), 119.2(\mathrm{CH}), 71.2(\mathrm{C}), 43.7(\mathrm{C}), 41.2\left(\mathrm{CH}_{2}\right), 33.9(\mathrm{CH})$, $33.7\left(\mathrm{CH}_{2}\right), 26.4\left(\mathrm{CH}_{2}\right), 21.2\left(\mathrm{CH}_{3}\right), 16.8\left(\mathrm{CH}_{3}\right)$. HRMS (ESIorbitrap) $m / z:[\mathrm{M}+\mathrm{Na}]^{+}$calcd for $\mathrm{C}_{14} \mathrm{H}_{16} \mathrm{~N}_{2} \mathrm{NaO}, 251.1155$; found, 251.1157

$\operatorname{rac}-(1 \mathrm{a} R, 9 \mathrm{~b} R, 9 \mathrm{c} R)-1,1 \mathrm{a}, 2,3,9 \mathrm{~b}, 9 \mathrm{c}-H e x a h y d r o-1-$ methylene-4H-cyclobuta $[h i]$ pyrido $[3,2-b]$ indolizin-4-one (2i): Yield: $27.8 \mathrm{mg}(0.131 \mathrm{mmol}, 66 \%)$ from $1 \mathbf{i}(42.3 \mathrm{mg}$, $0.199 \mathrm{mmol})$. Colorless solid. IR (KBr) 3065, 2952, 1686, 1597, 1412, 1368, 1324, 1221, 1199, 887, $785 \mathrm{~cm}^{-1} .{ }^{1} \mathrm{H}$ NMR $\left(400 \mathrm{MHz}, \mathrm{CDCl}_{3}\right) \delta 8.27$ (dd, $\left.J=5.3,1.7 \mathrm{~Hz}, 1 \mathrm{H}\right), 7.48$ (ddd, $J=7.3,1.6,0.6 \mathrm{~Hz}, 1 \mathrm{H}), 6.93(\mathrm{dd}, J=7.3,5.3 \mathrm{~Hz}, 1 \mathrm{H}), 5.03$ $5.01(\mathrm{~m}, 1 \mathrm{H}), 4.97-4.95(\mathrm{~m}, 1 \mathrm{H}), 4.76(\mathrm{t}, J=6.4 \mathrm{~Hz}, 1 \mathrm{H}), 4.24$ $4.21(\mathrm{~m}, 1 \mathrm{H}), 3.73-3.66(\mathrm{~m}, 1 \mathrm{H}), 2.60-2.54(\mathrm{~m}, 1 \mathrm{H}), 2.37-$ $2.24(\mathrm{~m}, 2 \mathrm{H}), 1.84-1.74(\mathrm{~m}, 1 \mathrm{H}) .{ }^{13} \mathrm{C}\left\{{ }^{1} \mathrm{H}\right\}$ NMR $(100 \mathrm{MHz}$, $\left.\mathrm{CDCl}_{3}\right) \delta 170.7(\mathrm{C}), 159.3(\mathrm{C}), 153.2(\mathrm{C}), 146.8(\mathrm{CH}), 131.7$ $(\mathrm{CH}), 127.1(\mathrm{C}), 118.9(\mathrm{CH}), 111.0\left(\mathrm{CH}_{2}\right), 58.8(\mathrm{CH}), 46.5$ $(\mathrm{CH}), 41.7(\mathrm{CH}), 35.1\left(\mathrm{CH}_{2}\right), 23.7\left(\mathrm{CH}_{2}\right)$. HRMS (ESIorbitrap) $m / z:[\mathrm{M}+\mathrm{Na}]^{+}$calcd for $\mathrm{C}_{13} \mathrm{H}_{12} \mathrm{~N}_{2} \mathrm{NaO}, 235.0842$; found, 235.0846

rac-(1aR,9bR,9cR)-1,1a,,2,3,9b,9c-Hexahydro-1,1dimethyl-4H-cyclobuta $[h i]$ pyrido $[3,2-b]$ indolizin-4-one

(2j): Yield: $34.8 \mathrm{mg}(0.152 \mathrm{mmol}, 75 \%)$ from $\mathbf{1 j}(46.5 \mathrm{mg}$, 0.204 mmol). Colorless oil. IR (KBr) 2952, 1691, 1597, 1456, 1411, 1369, 1329, 1218, $781 \mathrm{~cm}^{-1}$. ${ }^{1} \mathrm{H}$ NMR $(400 \mathrm{MHz}$, $\left.\mathrm{CDCl}_{3}\right) \delta 8.26(\mathrm{~d}, J=5.2 \mathrm{~Hz}, 1 \mathrm{H}), 7.37$ (dd, $\left.J=7.2,0.7 \mathrm{~Hz}, 1 \mathrm{H}\right)$, $6.92-6.88(\mathrm{~m}, 1 \mathrm{H}), 4.59(\mathrm{t}, J=6.8 \mathrm{~Hz}, 1 \mathrm{H}), 3.34(\mathrm{~d}, J=6.8 \mathrm{~Hz}$, $1 \mathrm{H}), 2.79\left(\mathrm{dt}, J_{\mathrm{d}}=10.7, J_{\mathrm{t}}=6.8 \mathrm{~Hz}, 1 \mathrm{H}\right), 2.54\left(\mathrm{dt}, J_{\mathrm{d}}=14.2\right.$, $\left.J_{\mathrm{t}}=2.5 \mathrm{~Hz}, 1 \mathrm{H}\right), 2.19\left(\mathrm{dt}, J_{\mathrm{d}}=5.5, J_{\mathrm{t}}=14.2 \mathrm{~Hz}, 1 \mathrm{H}\right), 2.08-2.00$ $(\mathrm{m}, 1 \mathrm{H}), 1.75-1.65(\mathrm{~m}, 1 \mathrm{H}), 1.30(\mathrm{~s}, 3 \mathrm{H}), 0.65(\mathrm{~s}, 3 \mathrm{H})$. ${ }^{13} \mathrm{C}\left\{{ }^{1} \mathrm{H}\right\}$ NMR $\left(100 \mathrm{MHz}, \mathrm{CDCl}_{3}\right) \delta 170.5(\mathrm{C}), 159.3(\mathrm{C})$, $146.7(\mathrm{CH}), 133.2(\mathrm{CH}), 126.5(\mathrm{C}), 118.6(\mathrm{CH}), 56.9(\mathrm{CH})$, $46.6(\mathrm{CH}), 41.6(\mathrm{C}), 40.9(\mathrm{CH}), 35.2\left(\mathrm{CH}_{2}\right), 32.2\left(\mathrm{CH}_{3}\right), 21.8$
$\left(\mathrm{CH}_{3}\right), 19.2\left(\mathrm{CH}_{2}\right)$. HRMS (ESI-orbitrap) $m / z:[\mathrm{M}+\mathrm{Na}]^{+}$calcd for $\mathrm{C}_{14} \mathrm{H}_{16} \mathrm{~N}_{2} \mathrm{NaO}$, 251.1155; found, 251.1157 .

\section{rac- $(4 \mathrm{~b} R, 4 \mathrm{c} S, 8 S, 8 \mathrm{a} R, 8 \mathrm{~b} R)-4 \mathrm{~b}, 4 \mathrm{c}, 5,6,7,8,8 \mathrm{a}, 8 \mathrm{~b}-$}

Octahydro-8,9-

ethanobenzo[3',4']cyclobuta[1',2':4,5]pyrrolo[2,3-

b]pyridin-10-one (2ka): Yield: $37.1 \mathrm{mg}$ (pure $1.7 \mathrm{mg}+35.4$ mg containing a small amount of AcOEt, $0.148 \mathrm{mmol}$ estimated by ${ }^{1} \mathrm{H}$ NMR, 68\%) from $1 \mathbf{k}(52.2 \mathrm{mg}, 0.217 \mathrm{mmol})$. Colorless oil. IR (KBr) 2941, 2866, 1677, 1595, 1406, 1351, 1332, 1291, 1181, 1087, 1035, $782 \mathrm{~cm}^{-1}$. ${ }^{1} \mathrm{H}$ NMR $(400 \mathrm{MHz}$, $\left.\mathrm{CDCl}_{3}\right) \delta 8.30(\mathrm{dd}, J=5.3,1.7 \mathrm{~Hz}, 1 \mathrm{H}), 7.45(\mathrm{dd}, J=7.3,1.7 \mathrm{~Hz}$, 1H), 6.96 (dd, $J=7.3,5.3 \mathrm{~Hz}, 1 \mathrm{H}), 4.78$ (t, $J=4.5 \mathrm{~Hz}, 1 \mathrm{H}), 3.36$ (dd, $J=8.0,4.5 \mathrm{~Hz}, 1 \mathrm{H}), 2.61-2.35(\mathrm{~m}, 5 \mathrm{H}), 1.75-1.34(\mathrm{~m}, 6 \mathrm{H})$. ${ }^{13} \mathrm{C}\left\{{ }^{1} \mathrm{H}\right\}$ NMR $\left(100 \mathrm{MHz}, \mathrm{CDCl}_{3}\right) \delta 171.9$ (C), 161.1 (C), $146.2(\mathrm{CH}), 131.2(\mathrm{CH}), 130.8(\mathrm{C}), 119.5(\mathrm{CH}), 63.6(\mathrm{CH})$, $46.6(\mathrm{CH}), 39.2(\mathrm{CH}), 38.0\left(\mathrm{CH}_{2}\right), 35.3(\mathrm{CH}), 30.0(\mathrm{CH}), 24.5$ $\left(\mathrm{CH}_{2}\right), 23.2\left(\mathrm{CH}_{2}\right), 19.6\left(\mathrm{CH}_{2}\right)$. HRMS (ESI-orbitrap) $\mathrm{m} / \mathrm{z}$ : [M $+\mathrm{Na}]^{+}$calcd for $\mathrm{C}_{15} \mathrm{H}_{16} \mathrm{~N}_{2} \mathrm{NaO}, 263.1155$; found, 263.1157 .

\section{rac-(4b $R, 4 \mathrm{c} S, 8 R, 8 \mathrm{a} R, 8 \mathrm{~b} R)-4 \mathrm{~b}, 4 \mathrm{c}, 5,6,7,8,8 \mathrm{a}, 8 \mathrm{~b}-$ Octahydro-8,9-}

ethanobenzo $\left[3^{\prime}, 4^{\prime}\right]$ cyclobuta[1',2':4,5]pyrrolo $[2,3-$

b]pyridin-10-one (2kb): Yield: $4.7 \mathrm{mg}(0.020 \mathrm{mmol}, 9 \%)$ from $1 \mathbf{k}(52.2 \mathrm{mg}, 0.217 \mathrm{mmol})$. Colorless oil. IR (KBr) 2931, $2858,1685,1596,1411,1375,1328,1305,1220,1118,1092$, $783 \mathrm{~cm}^{-1} .{ }^{1} \mathrm{H}$ NMR $\left(400 \mathrm{MHz}, \mathrm{CDCl}_{3}\right) \delta 8.30$ (dd, $J=5.2,1.6$ $\mathrm{Hz}, 1 \mathrm{H}), 7.39$ (dd, $J=7.3,1.6 \mathrm{~Hz}, 1 \mathrm{H}), 6.93$ (dd, $J=7.3,5.2 \mathrm{~Hz}$, $1 \mathrm{H}), 4.64-4.61(\mathrm{~m}, 1 \mathrm{H}), 3.89-3.85(\mathrm{~m}, 1 \mathrm{H}), 3.07-3.03(\mathrm{~m}$, $2 \mathrm{H}), 2.48-2.35(\mathrm{~m}, 3 \mathrm{H}), 1.83-1.76(\mathrm{~m}, 1 \mathrm{H}), 1.58-1.51(\mathrm{~m}$, $1 \mathrm{H}), 1.44-1.37(\mathrm{~m}, 1 \mathrm{H}), 1.08-0.92(\mathrm{~m}, 2 \mathrm{H}), 0.64-0.57(\mathrm{~m}$, 1H). ${ }^{13} \mathrm{C}\left\{{ }^{1} \mathrm{H}\right\}$ NMR $\left(100 \mathrm{MHz}, \mathrm{CDCl}_{3}\right) \delta 169.9(\mathrm{C}), 159.4(\mathrm{C})$, $146.5(\mathrm{CH}), 133.9(\mathrm{CH}), 126.6(\mathrm{C}), 118.8(\mathrm{CH}), 59.8(\mathrm{CH})$, $41.7\left(\mathrm{CH}_{2}\right), 39.5(\mathrm{CH}), 35.4(\mathrm{CH}), 30.3(\mathrm{CH}), 29.0\left(\mathrm{CH}_{2}\right), 27.6$ $(\mathrm{CH}), 24.5\left(\mathrm{CH}_{2}\right), 21.5\left(\mathrm{CH}_{2}\right)$. HRMS (ESI-orbitrap) $\mathrm{m} / \mathrm{z}$ : [M $+\mathrm{Na}]^{+}$calcd for $\mathrm{C}_{15} \mathrm{H}_{16} \mathrm{~N}_{2} \mathrm{NaO}, 263.1155$; found, 263.1158.

rac-(5a $R, 6 S)$-6-ethynyl-5a,6,7,8-tetrahydropyrido[3,2b]indolizin-9(5H)-one (4): Compound 4 was very hard to purify. It was isolated by HPLC after separation with preparative TLC. Conditions: column, COSMOSIL (Nacalai Tesque, 20 x $250 \mathrm{~mm}$ ); eluent: $\mathrm{CH}_{3} \mathrm{CN}: \mathrm{H}_{2} \mathrm{O}=40: 60$; flow, $5.7 \mathrm{mLmin}$ ${ }^{1}$; column temp, $40{ }^{\circ} \mathrm{C}$; detection, UV $254 \mathrm{~nm} ; t_{\mathrm{R}}=10.9 \mathrm{~min}$. This sample still contained a small amount of $\mathbf{2 i}$. Yield: 5.1 $\mathrm{mg}(0.024 \mathrm{mmol}, 12 \%)$ from $1 \mathbf{i}(42.3 \mathrm{mg}, 0.199 \mathrm{mmol})$. Colorless solid. IR (KBr) 3207, 3046, 2952, 2912, 2104, 1667, 1600, $1420,1391,1315,1237,805 \mathrm{~cm}^{-1}$. ${ }^{1} \mathrm{H}$ NMR $(400 \mathrm{MHz}$, $\left.\mathrm{CDCl}_{3}\right) \delta 8.33(\mathrm{~d}, J=5.2 \mathrm{~Hz}, 1 \mathrm{H}), 7.51(\mathrm{dd}, J=7.3,0.9 \mathrm{~Hz}, 1 \mathrm{H})$, $6.94(\mathrm{dd}, J=7.3,5.2 \mathrm{~Hz}, 1 \mathrm{H}), 4.36(\mathrm{ddd}, J=11.0,8.1,3.5 \mathrm{~Hz}$, $1 \mathrm{H}), 3.36$ (dd, $J=15.7,11.0 \mathrm{~Hz}, 1 \mathrm{H}), 3.22-3.19(\mathrm{~m}, 1 \mathrm{H}), 3.04$ (dd, $J=15.7,8.1 \mathrm{~Hz}, 1 \mathrm{H}), 2.92$ (ddd, $J=18.2,12.2,7.2 \mathrm{~Hz}, 1 \mathrm{H})$, 2.65 (ddd, $J=18.2,6.5,1.5 \mathrm{~Hz}, 1 \mathrm{H}), 2.25-2.19$ (m, $1 \mathrm{H}$, including $\mathrm{d}, J=2.4 \mathrm{~Hz}, 1 \mathrm{H}$ at 2.21$), 2.09-2.00(\mathrm{~m}, 1 \mathrm{H}) .{ }^{13} \mathrm{C}\left\{{ }^{1} \mathrm{H}\right\}$ NMR (100 MHz, $\left.\mathrm{CDCl}_{3}\right) \delta 167.1(\mathrm{C}), 156.5(\mathrm{C}), 147.1(\mathrm{C})$, $133.0(\mathrm{CH}), 123.8(\mathrm{C}), 119.0(\mathrm{CH}), 79.9(\mathrm{C}), 74.9(\mathrm{CH}), 61.7$ $(\mathrm{CH}), 31.3\left(\mathrm{CH}_{2}\right), 29.8(\mathrm{CH}), 29.7\left(\mathrm{CH}_{2}\right), 26.0\left(\mathrm{CH}_{2}\right)$. HRMS (ESI-orbitrap) $m / z:[\mathrm{M}+\mathrm{Na}]^{+}$calcd for $\mathrm{C}_{13} \mathrm{H}_{12} \mathrm{~N}_{2} \mathrm{NaO}$, 235.0842; found, 235.0846.

rac-(5a $R, 6 S)-6$-(prop-1-en-2-yl)-5a,6,7,8tetrahydropyrido[3,2-b]indolizin-9(5H)-one (5): Yield: 8.3 $\mathrm{mg}(0.036 \mathrm{mmol}, 18 \%)$ from $\mathbf{1 j}$ (46.5 mg, $0.204 \mathrm{mmol})$. Colorless oil. IR (KBr) 3071, 2944, 1677, 1597, 1422, 1392, 1299, 1240, 899, $788 \mathrm{~cm}^{-1} .{ }^{1} \mathrm{H}$ NMR $\left(400 \mathrm{MHz}, \mathrm{CDCl}_{3}\right) \delta 8.33(\mathrm{~d}$, $J=5.1 \mathrm{~Hz}, 1 \mathrm{H}), 7.49(\mathrm{dd}, J=7.3,1.1 \mathrm{~Hz}, 1 \mathrm{H}), 6.93(\mathrm{dd}, J=7.3$, $5.1 \mathrm{~Hz}, 1 \mathrm{H}), 4.96$ (t, $J=1.4 \mathrm{~Hz}, 1 \mathrm{H}), 4.74$ (br s, 1H), 4.52 (dt, 
$\left.J_{\mathrm{t}}=9.8 \mathrm{~Hz}, J_{\mathrm{d}}=4.7 \mathrm{~Hz}, 1 \mathrm{H}\right), 3.24(\mathrm{dd}, J=16.5,9.9 \mathrm{~Hz}, 1 \mathrm{H}), 3.02$ (dd, $J=16.5,9.6 \mathrm{~Hz}, 1 \mathrm{H}), 2.82-2.78(\mathrm{~m}, 1 \mathrm{H}), 2.69-2.53(\mathrm{~m}$, 2H), 2.19-2.10 (m, 1H), 2.03-1.95 (m, 1H), $1.69(\mathrm{~s}, 3 \mathrm{H})$. ${ }^{13} \mathrm{C}\left\{{ }^{1} \mathrm{H}\right\}$ NMR $\left(100 \mathrm{MHz}, \mathrm{CDCl}_{3}\right) \delta 168.5(\mathrm{C}), 156.1$ (C), $147.1(\mathrm{CH}), 142.6(\mathrm{C}), 133.3(\mathrm{CH}), 124.1(\mathrm{C}), 118.8(\mathrm{CH})$, $114.8\left(\mathrm{CH}_{2}\right), 60.6(\mathrm{CH}), 41.8(\mathrm{CH}), 31.2\left(\mathrm{CH}_{2}\right), 29.3\left(\mathrm{CH}_{2}\right)$, $24.5\left(\mathrm{CH}_{2}\right), 22.5\left(\mathrm{CH}_{3}\right)$. HRMS (ESI-orbitrap) $m / z$ : $[\mathrm{M}+\mathrm{Na}]^{+}$ calcd for $\mathrm{C}_{14} \mathrm{H}_{16} \mathrm{~N}_{2} \mathrm{NaO}$, 251.1155; found, 251.1158.

rac-Methyl 2-((4bR,4cS,8S,8aR,8bR)-4b,4c,6,7,8,8a,8b,9octahydro-5H-benzo[3',4']cyclobuta[1',2':4,5]pyrrolo[2,3b]pyridin-8-yl)acetate (6): Yield: $6.8 \mathrm{mg}(0.025 \mathrm{mmol}, 12 \%)$ from 1k (52.2 mg, $0.217 \mathrm{mmol})$. Colorless oil. IR (KBr) 3213, 2925, 2856, 1734, 1614, 1585, 1472, 1442, 1331, 1262, 1193, $1167,1017,772 \mathrm{~cm}^{-1} .{ }^{1} \mathrm{H}$ NMR $\left(400 \mathrm{MHz}, \mathrm{CDCl}_{3}\right) \delta 7.72(\mathrm{dd}$, $J=5.5,1.4 \mathrm{~Hz}, 1 \mathrm{H}), 7.16-7.14(\mathrm{~m}, 1 \mathrm{H}), 7.00(\mathrm{dd}, J=6.9,5.5 \mathrm{~Hz}$, 1H), 4.97 (br s, 1H), 4.68-4.67 (m, 1H), $3.71(\mathrm{~s}, 3 \mathrm{H}), 3.59(\mathrm{t}$, $J=6.7 \mathrm{~Hz}, 1 \mathrm{H}), 2.99$ (dd, $J=16.7,8.0 \mathrm{~Hz}, 1 \mathrm{H}), 2.51-2.49$ (br m, $1 \mathrm{H}), 2.43-2.25(\mathrm{~m}, 3 \mathrm{H}), 1.84-1.80(\mathrm{~m}, 1 \mathrm{H}), 1.62-1.48(\mathrm{~m}$, $3 \mathrm{H}), 1.44-1.24(\mathrm{~m}, 2 \mathrm{H}) .{ }^{13} \mathrm{C}\left\{{ }^{1} \mathrm{H}\right\} \mathrm{NMR}\left(100 \mathrm{MHz}, \mathrm{CDCl}_{3}\right) \delta$ $174.7(\mathrm{C}), 166.5(\mathrm{C}), 143.7(\mathrm{CH}), 129.8(\mathrm{CH}), 126.1(\mathrm{C})$, $112.8(\mathrm{CH}), 64.4(\mathrm{CH}), 51.6\left(\mathrm{CH}_{3}\right), 51.1(\mathrm{CH}), 48.7(\mathrm{CH})$, $42.7(\mathrm{CH}), 33.0\left(\mathrm{CH}_{2}\right), 32.1(\mathrm{CH}), 31.7\left(\mathrm{CH}_{2}\right), 31.0\left(\mathrm{CH}_{2}\right)$, $21.6\left(\mathrm{CH}_{2}\right)$. HRMS (ESI-orbitrap) $\mathrm{m} / \mathrm{z}$ : $[\mathrm{M}+\mathrm{Na}]^{+}$calcd for $\mathrm{C}_{16} \mathrm{H}_{21} \mathrm{~N}_{2} \mathrm{NaO}_{2}$, 273.1598; found, 273.1599.

rac-(3a $R, 4 \mathrm{a} R, 9 \mathrm{~b} R)-1,2,3,3 \mathrm{a}, 4,4 \mathrm{a}-h$ exahydro-5 $\mathrm{H}$ cyclopenta[1',4']cyclobuta[1',2':4,5]pyrrolo[2,3- $b]$ pyridine2,2,5-triicarboxylic acid 2,2-diethyl 5-methyl ester (8): Yield: $35.8 \mathrm{mg}$ (0.0922 mmol, 88\%) from 7 (40.5 mg, 0.104 mmol). Colorless oil. IR (KBr) 2980, 2953, 1730, 1702, 1599, $1585,1441,1422,1377,1337,1308,1254,1200,1142,1116$, $1088,1071,863,776,766 \mathrm{~cm}^{-1} .{ }^{1} \mathrm{H}$ NMR $\left(400 \mathrm{MHz}, \mathrm{CDCl}_{3}\right)$ $\delta 8.26(\mathrm{dd}, J=5.1,1.7 \mathrm{~Hz}, 1 \mathrm{H}), 7.51(\mathrm{dd}, J=7.4,1.7 \mathrm{~Hz}, 1 \mathrm{H})$, $6.91(\mathrm{dd}, J=7.4,5.1 \mathrm{~Hz}, 1 \mathrm{H}), 4.57$ (t, $J=5.9 \mathrm{~Hz}, 1 \mathrm{H}), 4.32$ (q, $J=7.1 \mathrm{~Hz}, 2 \mathrm{H}), 4.24$ (q, $J=7.1 \mathrm{~Hz}, 2 \mathrm{H}), 3.86$ (s, 3H), 2.88-2.82 (m, 1H), 2.79 (d, $J=14.3 \mathrm{~Hz}, 1 \mathrm{H}), 2.59$ (dd, $J=14.1,8.4 \mathrm{~Hz}$, $1 \mathrm{H}), 2.51$ (dd, $J=14.1,3.6 \mathrm{~Hz}, 1 \mathrm{H}), 2.49$ (d, $J=14.3 \mathrm{~Hz}, 1 \mathrm{H})$, 2.28-2.25 (m, 2H), $1.32(\mathrm{t}, J=7.1 \mathrm{~Hz}, 3 \mathrm{H}), 1.28(\mathrm{t}, J=7.1 \mathrm{~Hz}$, $3 \mathrm{H}) .{ }^{13} \mathrm{C}\left\{{ }^{1} \mathrm{H}\right\} \mathrm{NMR}\left(100 \mathrm{MHz}, \mathrm{CDCl}_{3}\right) \delta 171.5(\mathrm{C}), 171.4(\mathrm{C})$, $156.7(\mathrm{C}), 152.0(\mathrm{C}), 147.5(\mathrm{CH}), 131.6(\mathrm{CH}), 129.0(\mathrm{C})$, $118.1(\mathrm{CH}), 63.2(\mathrm{C}), 61.9\left(\mathrm{CH}_{2}\right), 61.8\left(\mathrm{CH}_{2}\right), 60.8(\mathrm{CH}), 54.7$ (C), $53.0\left(\mathrm{CH}_{3}\right), 45.8(\mathrm{CH}), 43.1\left(\mathrm{CH}_{2}\right), 40.7\left(\mathrm{CH}_{2}\right), 33.2$ $\left(\mathrm{CH}_{2}\right), 14.0\left(\mathrm{CH}_{3}\right)$. HRMS (ESI-orbitrap) $\mathrm{m} / z$ : $[\mathrm{M}+\mathrm{Na}]^{+}$calcd for $\mathrm{C}_{20} \mathrm{H}_{24} \mathrm{~N}_{2} \mathrm{NaO}_{6}, 411.1527$; found, 411.1525 .

\section{ASSOCIATED CONTENT}

\section{Supporting Information}

The Supporting Information is available free of charge on the ACS Publications website.

Figure S1-S3, Scheme S1, and spectral data of new compounds. (PDF) FAIR Data is available as Supporting Information for Publication and includes the primary NMR FID files for compounds 1a-1k, 2a-2j, 2ka, 2kb, 4-7.

\section{AUTHOR INFORMATION}

\section{Corresponding Authors}

Noriyoshi Arai - Division of Applied Chemistry, Faculty of Engineering, Hokkaido University, Sapporo, Hokkaido 060-8628,
Japan; orcid.org/0000-0003-0964-223X; E-mail: narai@eng.hokudai.ac.jp

Takeshi Ohkuma - Division of Applied Chemistry and Frontier Chemistry Center, Faculty of Engineering, Hokkaido University, Sapporo, Hokkaido 060-8628, Japan; orcid.org/0000-00025467-3169; E-mail: ohkuma@eng.hokudai.ac.jp

\section{Author Contributions}

The manuscript was written through a major contribution of N.A. Notes

The authors declare no competing financial interest.

\section{ACKNOWLEDGMENT}

We thank the Advanced Training Program for Catalytic Study, Institute for Catalysis, Hokkaido University, for providing computational resources. We are grateful to the Instrumental Analysis Support Office, the Frontier Chemistry Center, Faculty of Engineering, Hokkaido University for allowing us to use their NMR spectrometer for the compound analyses. Finally, we would like to express our gratitude to the Instrumental Analysis Division, Global Facility Center, Creative Research Institution, Hokkaido University for performing the mass spectrometry and elemental analyses and providing insight and expertise that greatly assisted our research.

\section{REFERENCES}

(1) Reviews: (a) Irie, T.; Sawa, M. 7-Azaindole: A Versatile Scaffold for Developing Kinase Inhibitors. Chem. Pharm. Bull. 2018, 66, 29-36. (b) Popowycz, F.; Routier, S.; Joseph, B.; and Mérour, J.-Y. Synthesis and reactivity of 7-azaindole (1H-pyrrolo[2,3-b]pyridine). Tetrahedron 2007, 63, 1031-1064.

(2) Nuhant, P.; Allais, C.; Chen, M. Z.; Coe, J. W.; Dermenci, A.; Fadeyi, O. O.; Flick, A. C.; Mousseau, J. J. Access to Highly Substituted 7-Azaindoles from 2-Fluoropyridines via 7-Azaindoline Intermediates. Org. Lett. 2015, 17, 4292-4295.

(3) Takai, K.; Inoue, Y.; Konishi, Y.; Suwa, A.; Uruno, Y.; Matsuda, H.; Nakako, T.; Sakai, M.; Nishikawa, H.; Hashimoto, G.; Enomoto, T.; Kitamura, A.; Uematsu, Y.; Kiyoshi, A.; Sumiyoshi, T. Discovery of $\mathrm{N}$-Substituted 7-Azaindoline Derivatives as Potent, Orally Available $\mathrm{M}_{1}$ and $\mathrm{M}_{4}$ Muscarinic Acetylcholine Receptors Selective Agonists. Bioorg. Med. Chem. Lett. 2014, 24, 3189-3193.

(4) Badarau, E.; Putey, A.; Suzenet, F.; Joseph, B.; Bojarski, A.; Finaru, A.; Guillaumet, G. New Insights into Homopiperazine-based 5- $\mathrm{HT}_{1 \mathrm{~A}} / 5-\mathrm{HT}_{7} \mathrm{R}$ Ligands: Synthesis and Biological Evaluation. $J$. Enzyme Inhib. Med. Chem. 2010, 25, 301-305.

(5) Bristow, L. J.; Kramer, M. S.; Kulagowski, J.; Patel, S.; Ragan, C. I.; Seabrook, G. R. Schizophrenia and L-745, 870, a Novel Dopamine D4 Receptor Antagonist. Trends Pharnacol. Sci. 1997, 18, 186188.

(6) (a) Lovering, F. Escape from Flatland 2: Complexity and Promiscuity. MedChemComm 2013, 4, 515-519. (b) Lovering, F.; Bikker, J.; Humblet, C. Escape from Flatland: Increasing Saturation as an Approach to Improving Clinical Success. J. Med. Chem. 2009, 52, 6752-6756.

(7) Stepan, A. F.; Subramanyam, C.; Efremov, I. V.; Dutra, J. K.; O'Sullivan, T. J.; DiRico, K. J.; McDonald, W. S.; Won, A.; Dorff, P. H.; Nolan, C. E.; Becker, S. L.; Pustilnik, L. R.; Riddell, D. R.; Kauffman, G. W.; Kormos, B. L.; Zhang, L.; Lu, Y.; Capetta, S. H.; Green, M. E.; Karki, K.; Sibley, E.; Atchison, K. P.; Hallgren, A. J.; Oborski, C. E.; Robshaw, A. E.; Sneed, B.; O’Donnell, C. J. Application of the Bicyclo[1.1.1]pentane Motif as a Nonclassical Phenyl Ring Bioisostere in the Design of a Potent and Orally Active $\gamma$-Secretase Inhibitor. J. Med. Chem. 2012, 55, 3414-3424.

(8) Levterov, V. V.; Panasyuk, Y.; Pivnytska, V. O.; Mykhailiuk, P. K. Water-Soluble Non-Classical Benzene Mimetics. Angew. Chem. Int. Ed. 2020, 59, 7161-7167.

(9) (a) Oderinde, M. S.; Mao, E.; Ramirez, A.; Pawluczyk, J.; Jorge, C.; Cornelius, L. A. M.; Kempson, J.; Vetrichelvan, M.; Pitchai, M.; 
Gupta, A.; Gupta, A. K.; Meanwell, N. A.; Mathur, A.; Murali Dhar, T. G. Synthesis of Cyclobutane-Fused Tetracyclic Scaffolds via Visible-Light Photocatalysis for Building Molecular Complexity. J. Am. Chem. Soc. 2020, 142, 3094-3103. (b) Zhu, M.; Zheng, C.; Zhang, X.; You, S.-L. Synthesis of Cyclobutane-Fused Angular Tetracyclic Spiroindolines via Visible-Light-Promoted Intramolecular Dearomatization of Indole Derivatives. J. Am. Chem. Soc. 2019, 141, 26362644. (c) Levterov, V. V.; Michurin, O.; Borysko, P. O.; Zozulya, S.; Sadkova, I. V.; Andrey A. Tolmachev, A. A.; Mykhailiuk, P. K. Photochemical In-Flow Synthesis of 2,4-Methanopyrrolidines: Pyrrolidine Analogues with Improved Water Solubility and Reduced Lipophilicity. J. Org. Chem. 2018, 83, 14350-14361. (d) Druzhenko, T.; Skalenko, Y.; Samoilenko, M.; Denisenko, A.; Zozulya, S.; Borysko, P. O.; Sokolenko, M. I.; Tarasov, A.; Mykhailiuk, P. K. Photochemical Synthesis of 2-Azabicyclo[3.2.0]heptanes: Advanced Building Blocks for Drug Discovery. Synthesis of 2,3-Ethanoproline. J. Org. Chem. 2018, 83, 1394-1401. (e) Nandi, R. K.; Guillot, R.; Kouklovsky, C.; Vincent, G. Synthesis of 3,3-Spiroindolines via $\mathrm{FeCl}_{3}$-Mediated Cyclization of Aryl- or Alkene-Containing 3-Substituted N-Ac Indoles. Org. Lett. 2016, 18, 1716-1719. (f) Blanco-Ania, D.; Gawade, S. A.; Zwinkels, L. J. L.; Maartense, L.; Bolster, M. G.; Benningshof, J. C. J.; Rutjes, F. P. J. T. Rapid and Scalable Access into Strained Scaffolds through Continuous Flow Photochemistry. Org. Process Res. Dev. 2016, 20, 409-413. (g) Martin, V. I.; Goodell, J. R.; Ingham, O. J.; Porco, Jr., J. A.; Beeler, A. B. Multidimensional Reaction Screening for Photochemical Transformations as a Tool for Discovering New Chemotypes. J. Org. Chem. 2014, 79, 3838-3846.

(10) Reviews: (a) Poplata, S.; Tröster, A.; Zou, Y.-Q.; Bach, T. Recent Advances in the Synthesis of Cyclobutanes by Olefin [2+2] Photocycloaddition Reactions. Chem. Rev. 2016, 116, 9748-9815. (b) Bach, T.; Hern, J. P. Photochemical Reactions as Key Steps in Natural Product Synthesis. Angew. Chem. Int. Ed. 2011, 50, 1000-1045. (c) Hoffmann, N. Chem. Rev. 2008, 108, 1052-1103. (d) Hehn, J. P.; Müller, C.; Bach, T. In Handbook of Synthetic Photochemistry; Albini, A., Fagnoni, M., Eds.; Wiley-VCH: Weinheim, Germany, 2010; pp 171-215. (e) Hoffmann, N. In Handbook of Synthetic Photochemistry; Albini, A., Fagnoni, M., Eds.; Wiley-VCH: Weinheim, Germany, 2010; pp 137-169. (f) Fleming, S. A.; Bradford, C. L.; Gao, J. J. In Organic Photochemistry; Ramamurthy, V., Schanze, K. S., Eds.; Marcel Dekker: New York, 1997; pp 187-243. (g) Crimmins, M. T.; Reinhold, T. L. Enone Olefin [2+2] Photochemical Cycloadditions. Org. React. 1993, 44, 297-588.

(11) (a) Arai, N.; Mizota, M.; Ohkuma, T. Stereoselective Preparation of Spiro[4.4] Cyclic Compounds by the Photochemical Activation of Oxazoles. Org. Lett. 2015, 17, 86-89. (b) Arai, N.; Mizota, M.; Ohkuma, T. Novel Intramolecular Cyclization-Skeletal Reorganization of 2-Arylthiazoles under Photoirradiation. Heterocycles 2015 , 90,607-616. (c) Arai, N.; Tanaka, K.; Ohkuma, T. Novel Intramolecular Photocyclization of $\alpha$-Arylthiophene Derivatives. Org. Lett. 2012, 14, 1488-1491. (d) Arai, N.; Tanaka, K.; Ohkuma, T. Photosensitized Intramolecular Cyclization of Furan and Non-activated Alkene: Pathway Switching by the Substituent on the Furan Ring. Tetrahedron Lett. 2010, 51, 1273-1275.

(12) (a) Arai, N.; Ohkuma, T. Stereoselective Construction of Methylenecyclobutane-Fused Indolines through Photosensitized [2+2] Cycloaddition of Allene-Tethered Indole Derivatives. Org. Lett. 2019, 21, 1506-1510. (b) Arai, N.; Ohkuma, T. Stereoselective Preparation of Methylenecyclobutane-fused Angular Tetracyclic Spiroindolines via Photosensitized Intramolecular [2+2] Cycloaddition with Allene. Tetrahedron Lett. 2019, 60, 151252.

(13) Nakano, T.; Santana. M. Photoaddition of Benzophenone to Azaindole, Synthesis of the Oxetane of 7-Azaindole. J. Heterocycl. Chem. 1976, 13, 585-587.

(14) Helgen, C.; Bochet, C. G. Pyridine-derived Heterocycles as Potential Photoacylating Reagents. Heterocycles 2006, 67, 797-805.

(15) Paternò-Büchi type oxetane formation of indoles. (a) Mateos, J.; Vega-Peñaloza, A.; Franceschi, P.; Rigodanza, F.; Andreetta, P.; Companyó, X.; Pelosi, G.; Marcella Bonchio, M.; Dell'Amico, L. A Visible-light Paternò-Büchi Dearomatisation Process towards the Construction of Oxetoindolinic Polycycles. Chem. Sci. 2020, 11,
6532-6538. (b) Franceschi, P.; Mateos, J. Vega-Peñaloza, A.; Dell'Amico, L. Microfluidic Visible-Light Paternò-Büchi Reaction of Oxindole Enol Ethers. Eur. J. Org. Chem. 2020, DOI: 10.1002/ejoc.202001057.

(16) Zhu, M.; Huang, X.-L.; Xu, H.; Zhang, X.; Zheng, C.; You, S.-L. Visible-Light-Mediated Synthesis of Cyclobutene-Fused Indolizidines and Related Structural Analogs. CCS Chem. 2020, 2, 652 664.

(17) Zhang, Z.; Yi, D.; Zhang, M.; Wei, J.; Lu, J.; Yang, L.; Wang, J.; Hao, N.; Pan, X.; Zhang, S.; Wei, S.; Fu, Q. Photocatalytic Intramolecular [2 +2$]$ Cycloaddition of Indole Derivatives via Energy Transfer: A Method for Late-Stage Skeletal Transformation. ACS Catal. 2020, 10, 10149-10156.

(18) The preparation of cyclobutane-fused pyrrolo[2,3- $b]$ pyridine derivatives through enamine formation/electrocyclization sequence was previously reported. Walter, H.; Sundermann, C. Novel Complex $\mathrm{N}$-Heterocycles via Intramolecular 1,5-Electrocyclizations: $1,2,4,4 \mathrm{a}, 5,5 \mathrm{a}, 10-$

Octahydropyrido[4", 3":2', 3']cyclobuta[1',2':4,5]pyrrolo[2,3b]pyridines. Heterocycles 1998, 48, 1581-1591.

(19) Quite recently, a photochemical dearomative [2+2] cycloaddition/ring-expansion sequence with indoles catalyzed by $\mathrm{Gd}(\mathrm{OTf})_{3}$ has been reported. Ma, J.; Schäfers, F.; Daniliuc, C.; Bergander, K.; Strassert, C. A.; Glorius, F. Gadolinium Photocatalysis: Dearomative [2+2] Cycloaddition/Ring-Expansion Sequence with Indoles. Angew. Chem. Int. Ed. 2020, 59, 9639-9645.

(20) (a) Carruthers, W.; Evans, N. Photolysis of 1-Benzoyl- and 1and 3-o-Iodobenzoyl-indole. J. Chem. Soc., Perkin Trans. 1 1974, 1523-1525. (b) Somei, M.; Natsume, M. Photochemical Rearrangements for the Syntheses of 3-, 4-, and 6-Substituted Indoles. Tetrahedron Lett. 1973, 2451-2454.

(21) The ground states of $\mathbf{1 a}$ and the corresponding indole derivative were optimized using B3LYP/6-311++G(2d,p) with the SMD model (acetone) in Gaussian 09. Gaussian 09, Revision D.01, Frisch, M. J.; Trucks, G. W.; Schlegel, H. B.; Scuseria, G. E.; Robb, M. A.; Cheeseman, J. R.; Scalmani, G.; Barone, V.; Petersson, G. A.; Nakatsuji, H.; Li, X.; Caricato, M.; Marenich, A.; Bloino, J.; Janesko, B. G.; Gomperts, R.; Mennucci, B.; Hratchian, H. P.; Ortiz, J. V.; Izmaylov, A. F.; Sonnenberg, J. L.; Williams-Young, D.; Ding, F.; Lipparini, F.; Egidi, F.; Goings, J.; Peng, B.; Petrone, A.; Henderson, T.; Ranasinghe, D.; Zakrzewski, V. G.; Gao, J.; Rega, N.; Zheng, G.; Liang, W.; Hada, M.; Ehara, M.; Toyota, K.; Fukuda, R.; Hasegawa, J.; Ishida, M.; Nakajima, T.; Honda, Y.; Kitao, O.; Nakai, H.; Vreven, T.; Throssell, K.; Montgomery, Jr., J. A.; Peralta, J. E.; Ogliaro, F.; Bearpark, M.; Heyd, J. J.; Brothers, E.; Kudin, K. N.; Staroverov, V. N.; Keith, T.; Kobayashi, R.; Normand, J.; Raghavachari, K.; Rendell, A.; Burant, J. C.; Iyengar, S. S.; Tomasi, J.; Cossi, M.; Millam, J. M.; Klene, M.; Adamo, C.; Cammi, R.; Ochterski, J. W.; Martin, R. L.; Morokuma, K.; Farkas, O.; Foresman, J. B.; Fox, D. J., Gaussian, Inc., Wallingford CT, 2016.

(22) Chromophore activation in the presence of Lewis acids. (a) Brenninger, C.; Jolliffe, J. D.; Bach, T. Chromophore Activation of $\alpha, \beta$-Unsaturated Carbonyl Compounds and Its Application to Enantioselective Photochemical Reactions. Angew. Chem. Int. Ed. 2018, 57, 14338-14349. (b) Blum, T. R.; Miller, Z. D.; Bates, D. M.; Guzei, I. A.; Yoon, T. P. Enantioselective Photochemistry through Lewis Acid-catalyzed Triplet Energy Transfer. Science 2016, 354, 1391-1395.

(23) Lathioor, E. C.; Leigh, W. J. Bimolecular Hydrogen Abstraction from Phenols byAromatic Ketone Triplets. Photochem. Photobiol. 2006, 82, 291-300.

(24) (a) Weedon, A. C.; Zhang, B. Removable Groups for Activation of Indole Photochemistry. Synthesis 1992, 95-100. (b) Hastings, D. J.; Weedon, A. C. Structures and Lifetimes of 1,4-Biradical Intermediates in the Photochemical Cycloaddition Reactions of $\mathrm{N}$ Benzoylindole with Alkenes. J. Org. Chem. 1991, 56, 6326-6331. (c) Hastings, D. J.; Weedon, A. C. The Origin of the Regioselectivity in the 2+2 Photochemical Cycloaddition Reactions of $N$-Benzoylindole with Alkenes: Trapping of 1,4-Biradical Intermediates with Hydrogen Selenide. Tetrahedron Lett. 1991, 32, 4107-4110. (d) Oldroyd, D. L.; Weedon, A. C. Solvent- and Wavwlength-dependent Photochemistry of $\mathrm{N}$-Benzoylindole and $\mathrm{N}$-Ethoxycarbonylindole. J. Photochem. 
Photobiol. A: Chem. 1991, 57, 207-216. (e) Hastings, D. J.; Weedon, A. C. Stereochemical Studies of the Photochemical Cycloaddition Reaction of Alkenes with $\mathrm{N}$-Benzoylindole and $\mathrm{N}$-Carbethoxyindole; Evidence for Biradical Intermediacy. Can. J. Chem. 1991, 69, 11711181. (f) Disanayaka, B. W.; Weedon, A. C. The Mechanism of the Photochemical Cycloaddition Reaction of $\mathrm{N}$-Benzoylindole with Cyclopentene. Can. J. Chem. 1990, 68, 1685-1692.

(25) (a) Oldroyd, D. L.; Weedon, A. C. Intramolecular Photochemical Cycloaddition Reactions of $N$-[( $\omega$-Alkenyloxy $)$ carbonyl $]$ indoles and $N$-( $\omega$-Alkenoyl)indoles. J. Org. Chem. 1994, 59, 1333-1343. (b) Oldroyd, D. L.; Weedon, A. C. Intramolecular Photochemical Cycloaddition Reactions of $\mathrm{N}$-Alkenyloxycarbonylindoles and $\mathrm{N}$ Alkenoylindoles. J. Chem. Soc., Chem. Commun. 1992, 1491-1492.

(26) Umehara, A.; Ueda, H.; Tokuyama, H. Condensation of Carboxylic Acids with Non-Nucleophilic $\mathrm{N}$-Heterocycles and Anilides Using $\mathrm{Boc}_{2} \mathrm{O}$. J. Org. Chem. 2016, 81, 11444-11453.

(27) (a) Trost, B. M.; McClory, A. Ruthenium-Catalyzed Alkylative Lactonization and Carbocyclization. Org. Lett. 2006, 8, 36273629. (b) Searles, S.; Li, Y.; Nassim, B.; Lopes, M.-T. R.; Tran, P. T.;
Crabbé, P. Observation on the Synthesis of Allenes by Homologation of Alk-1-ynes. J. Chem. Soc., Perkin Trans. 1 1984, 747-751.

(28) Shuler, S. A.; Yin. G.; Krause, S. B.; Vesper, C. M.; Watson, D. A. Synthesis of Secondary Unsaturated Lactams via an Aza-Heck Reaction. J. Am. Chem. Soc. 2016, 138, 13830-13833. 